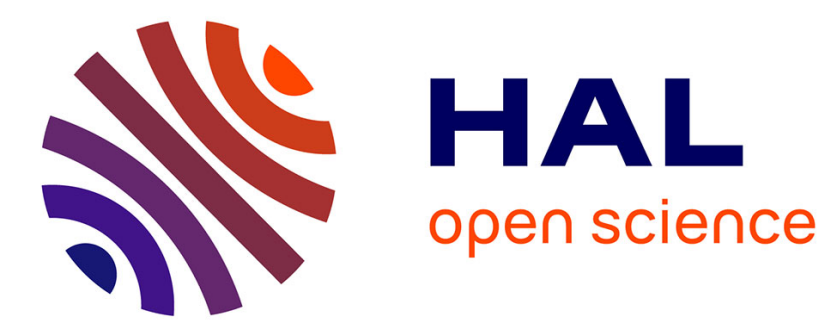

\title{
Low-Speed Turbofan Aerodynamic and Acoustic Prediction with an Isothermal Lattice Boltzmann Method
}

\author{
Majd Daroukh, Thomas Le Garrec, Cyril Polacsek
}

\section{To cite this version:}

Majd Daroukh, Thomas Le Garrec, Cyril Polacsek. Low-Speed Turbofan Aerodynamic and Acoustic Prediction with an Isothermal Lattice Boltzmann Method. AIAA Journal, 2021. hal-03368520

\author{
HAL Id: hal-03368520 \\ https://hal.science/hal-03368520
}

Submitted on 6 Oct 2021

HAL is a multi-disciplinary open access archive for the deposit and dissemination of scientific research documents, whether they are published or not. The documents may come from teaching and research institutions in France or abroad, or from public or private research centers.
L'archive ouverte pluridisciplinaire HAL, est destinée au dépôt et à la diffusion de documents scientifiques de niveau recherche, publiés ou non, émanant des établissements d'enseignement et de recherche français ou étrangers, des laboratoires publics ou privés. 


\title{
Low-Speed Turbofan Aerodynamic and Acoustic Prediction with an Isothermal Lattice Boltzmann Method
}

\author{
Majd Daroukh*, Thomas Le Garrec ${ }^{\dagger}$, Cyril Polacsek ${ }^{\ddagger}$ \\ DAAA, ONERA, Paris-Saclay University \\ F-92322 Châtillon - France
}

The objective of the paper is to assess the capability of the isothermal Lattice Boltzmann Method (LBM) to correctly capture aerodynamic and acoustic features from a low-speed turbofan. The evaluation is done on the Advanced Noise Control Fan (ANCF) model developed by NASA Glenn Research Center. An extensive comparison of the measured and computed aerodynamic results is presented for the first time on this configuration. The trends are well captured but quantitative discrepancies are observed for some variables, leading to lower fan performance values in the simulations. This last point was also observed in the previous related studies but remained unexplained. By validating in parallel the LBM aerodynamic results with a Reynolds-Averaged Navier-Stokes (RANS) simulation provided by the database, a likely uncertainty of the absolute values of some variables in the experimental mean flow data is shown (a result of the intent that experimental data was not acquired with absolute levels as an objective). Direct in-duct and far-field acoustic results are also investigated, taking advantage of the low-dissipative characteristics of the LBM. A good agreement with the measurements in terms of broadband noise is obtained, but the low frequencies tend to be overestimated. For the tonal noise, if the expected acoustic modes are recovered, quantitative differences are observed. Predictions based on an hybrid method where the acoustic sources computed by the LBM are propagated analytically using Goldstein's analogy are also made and compare well with the direct predictions, thus proving the correct propagation of acoustic waves by the solver.

\section{Introduction}

The Lattice Boltzmann Method (LBM) has emerged a few decades ago as an efficient alternative way to deal with Computational Fluid Dynamics (CFD) [1-9]. The study of complex fluid dynamic problems is usually done by solving the Navier-Stokes (NS) equations which describe the conservation of macroscopic quantities representing the fluid (mass, momentum and energy typically). The LBM relies on a different philosophy where the fluid is described by probability distribution functions representing discrete fluid particles (mesoscopic approach) [10]. The distribution

\footnotetext{
*Research Engineer, Department of Aerodynamics, Aeroelasticity, and Aeroacoustics, majd.daroukh@onera.fr.

${ }^{\dagger}$ Research Engineer, Department of Aerodynamics, Aeroelasticity, and Aeroacoustics, thomas.le_garrec@onera.fr.

${ }_{\ddagger}^{\ddagger}$ Research Engineer, Department of Aerodynamics, Aeroelasticity, and Aeroacoustics, cyril.polacsek@onera.fr.
} 
functions are classically governed by a simple stream and collide equation. The streaming process occurs in a discrete velocity space, defining the lattice, such that the distance between two defined points in each velocity direction is covered during one time step. The streaming operation is exact, extremely simple from a computational point of view and non-dissipative (but dissipation may be introduced by other terms). Adding the fact that the collision term is local, the LBM is particularly efficient and suited for massively parallel computations. However, the method has some drawbacks. First, the lattice imposes the space discretization in the form of an uniform Cartesian grid. Although it considerably simplifies the numerical setup by removing the meshing step, it also introduces difficulties 1) at the boundaries where immersed boundary conditions are needed for curved geometries and 2) at the mesh transitions (required to introduce local mesh refinements with a controlled total number of nodes) where the mesh cells must be twice bigger in each direction to respect the lattice [11]. In addition, the discretization of velocity space can introduce an error in the recovered NS equations by a Chapman-Enskog expansion. For quasi-incompressible flows, a 19-velocity lattice is often used in three dimensions (D3Q19 scheme) and allows the athermal formulation of the NS equations to be recovered with an error that scales with the Mach number raised to the third power [12]. In that case, the temperature cannot vary and the method is called isothermal LBM. For high Mach number values, a specific treatment is needed (increase of the size of the lattice, use of a double distribution function or hybrid method by coupling with the energy conservation equation solved in finite volume for instance) and the method is often referred to as thermal LBM. This treatment increases the complexity of the method and the computational ressources needed to solve a problem. In practice, the LBM solvers therefore include several formulations depending on the maximum expected Mach number.

Turbomachinery applications using an LBM solver started to be studied since 2012 [4, 9, 13,-16]. To the authors knowledge, only results obtained with the PowerFLOW code have been published up to now. Two configurations, the Advanced Noise Control Fan (ANCF) [17] and the Source Diagnostic Test (SDT) [18] from NASA and NASA/GE respectively, have been particularly investigated. The NASA Glenn Research Center provided many experimental data for both configurations thus allowing for an advanced validation of aerodynamic and acoustic features. The first studies are dedicated to the ANCF configuration because its low fan-blade tip Mach number at nominal speed (0.34) is compatible with the use of an isothermal LBM (D3Q19 scheme) [13, 15]. The aerodynamics is not deeply investigated in these studies, despite some assessment of the total pressure and velocity radial profiles in the inlet and some considerations about the mass flow rate. The latter is surprisingly underestimated by $10 \%$ to $15 \%$ in the simulations, which is far above the commonly accepted error for performance prediction (around 1\%). However, the acoustics has been much more reviewed using both direct predictions and hybrid predictions based on Ffowcs Williams and Hawkings analogy [19]. The broadband noise is quite well reproduced in the study of Mann et al. [13] but tends to be underestimated by 5 to $10 \mathrm{~dB}$ in the one of Orselli et al. [15]. In both studies, the tonal noise is importantly overestimated with gaps of 5 to $20 \mathrm{~dB}$ at the Blade Passing Frequency (BPF) and its first two harmonics. However, the shape of the directivity is similar to the experimental one and the emergence of the expected interaction modes is well predicted. For the SDT 
fan, the simulation is more complex because of the high subsonic (at approach and cutback) or transonic (at sideline) fan-blade tip speed that makes the use of a thermal LBM mandatory. In the related studies, an hybrid method that couples a D3Q19 scheme with the solution of the entropy equation through finite difference scheme is used for the high subsonic operating points [4]. For the transonic case, a different strategy is adopted with the coupling between a D3Q19 scheme with a D3Q39 scheme, the latter being activated only in the volume past the fan where transonic speeds are expected [16]. Even if the studies started later than on the ANCF case, they have been more thoroughly investigated with validation of both aerodynamics and acoustics. The acoustics is very well reproduced with differences of 1 to $2 \mathrm{~dB}$ on broadband noise levels and about $3 \mathrm{~dB}$ on tonal noise levels.

The present paper is limited to the isothermal LBM, and the objective is to evaluate its capabilities to correctly predict both the aerodynamic and acoustic features of a low-subsonic turbofan. Thermal LBM could of course be used for such turbofans but it will inevitably be more demanding in terms of complexity and computational resources. There is therefore an interest to keep using an isothermal formulation of the LBM whenever possible. For this evaluation, the ANCF test case is the perfect candidate because many instrumentations are included in the test bed, providing measurements of aerodynamic profiles inside the duct, unsteady loadings on the stator vanes and far-field noise. There are even the results of a Reynolds-Averaged Navier-Stokes (RANS) simulation in the database, which might also be used for aerodynamic validation as it is a robust and mature technique for mean flow prediction. Compared to the previous studies on the ANCF configuration [13, 15], a deeper analysis on aerodynamics will be provided. Indeed, if the generation and convection of turbulent structures and the propagation of acoustic waves are not expected to be affected by the isothermal assumption, the effect on the mean aerodynamics is less clear. In particular, a turbofan creates a pressure rise and a temperature rise across it but the latter is restrained in the isothermal formulation. The direct consequence is that the fan efficiency, defined as a function of the temperature rise, cannot be predicted by the method. However, the effect on other fan performance coefficients, such as the mass flow rate or the pressure ratio, and on the mean flow profiles inside the duct is not obvious. This is why all these characteristics should be evaluated before analyzing the acoustic results. The paper is structured as follows. The ANCF model is first described in Sec. II before detailing the numerical methodology based on LBM simulations with the ProLB solver in Sec. IIII. The aerodynamic results are then discussed in Sec. IV and acoustic predictions are carefully analyzed in Sec. V $\mathrm{V}$ A conclusion finally provides the main findings of the paper.

\section{The Advanced Noise Control Fan Model}

The ANCF test bed, located in the Aero-Acoustic Propulsion Laboratory (AAPL) at NASA Glenn Research Center shown in Fig. 1, was developed in the early 1990s to evaluate fan noise reduction concepts and to provide a database for code verification [17, 20-22]. It consists of a 16-blade ducted fan with a diameter of about $1.22 \mathrm{~m}$. The pitch of the blades can vary from 18 degrees to 38 degrees, the nominal value being 28 degrees. The nominal operating condition is 
$1800 \mathrm{rpm}$ resulting in a tip Mach number of about 0.34, an inlet duct Mach number around 0.15 and a Blade Passing Frequency $(\mathrm{BPF})$ of $480 \mathrm{~Hz}$. The fan speed can be varied from 100 to $2400 \mathrm{rpm}$. The ANCF can be run rotor alone or with a stator with variable spacing (typically half a rotor chord or one rotor chord) and vane counts $(13,14,26$ or 28 vanes).

Aerodynamic and acoustic measurements are available for the different configurations but are most complete at nominal speed $(1800 \mathrm{rpm})$, either rotor alone, or with 14 stator vanes spaced from the rotor by one rotor chord (written $c_{R}$ and defined from the rotor trailing edge to the stator leading edge at hub). Steady pressure probes and hotfilm traverses provide aerodynamic profiles upstream and downstream of the rotor for these cases. For the 14 stator vane configurations, measurements of the vane unsteady pressures are available and allow for an evaluation of the acoustic sources linked to rotor-stator interaction. All the configurations are otherwise instrumented for evaluation of acoustic propagation and radiation with duct modes measurements and far-field microphones. The in-duct acoustic modes are evaluated at inlet and outlet through a rotating rake and the 30 far-field microphones are placed on an arc with a radius of about $3.66 \mathrm{~m}$ from the inlet and exhaust exit planes. More details on the experimental setup and the available measurements can be found in the paper of McAllister et al. [22].

Note that RANS results are also provided in the database transferred by the NASA. The corresponding operating condition is $2000 \mathrm{rpm}$ and the simulated stator is equipped with 14 vanes and is spaced from the rotor by $0.27 c_{R}$.

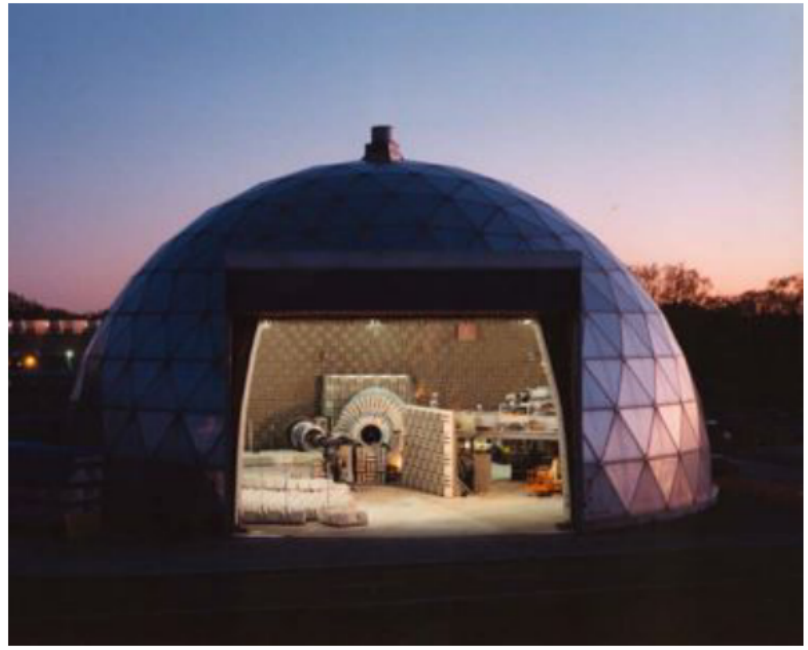

(a) The Aero-Acoustic Propulsion Laboratory (AAPL).

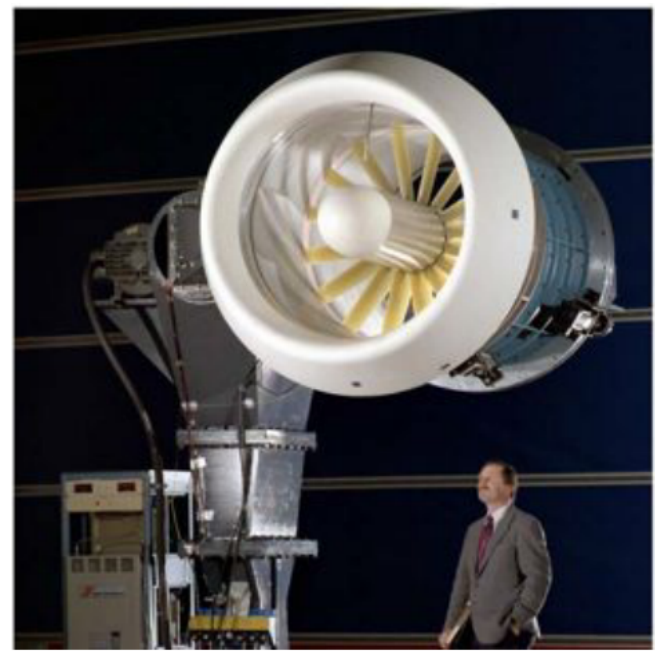

(b) The Advanced Noise Control Fan (ANCF).

Fig. 1 Pictures of the test bed. Reprinted from the paper of Loew et al. [17]. 


\section{Numerical Methodology}

\section{A. The ProLB Solver}

The ProLB solver used in this study is developed in the framework of a French consortium composed of universities, research institutes and industries (http://www.prolb-cfd.com/). Unlike standard CFD solver where the NavierStokes equations are solved, the governing equation here is the Boltzmann equation:

$$
\frac{\partial f(\boldsymbol{x}, t, \boldsymbol{\xi})}{\partial t}+\xi_{i} \frac{\partial f(\boldsymbol{x}, t, \boldsymbol{\xi})}{\partial x_{i}}=\left(\frac{\partial f(\boldsymbol{x}, t, \boldsymbol{\xi})}{\partial t}\right)_{\text {coll }}
$$

$f(\boldsymbol{x}, t, \boldsymbol{\xi})$ is the distribution function which represents the density of particles at point $\boldsymbol{x}$, time $t$ and with a velocity $\boldsymbol{\xi}$. The term on the right-hand side is the collision term that describes the variation rate of energy redistribution between particles. All the physics is included through the modeling of this term. The H-theorem dictates that the collision term increases the entropy, which means that the distribution function tends towards an equilibrium. This equilibrium is described by the Maxwell-Boltzmann distribution:

$$
f^{\mathrm{eq}}(\boldsymbol{x}, t, \boldsymbol{\xi})=\rho\left(\frac{m}{2 \pi k_{B} T}\right)^{D / 2} \exp \left(\frac{-m|\boldsymbol{\xi}-\boldsymbol{u}|^{2}}{2 k_{B} T}\right)
$$

with $m$ the particle mass, $k_{B}$ the Boltzmann constant, $D$ the degree of freedom of the particle, $\rho$ the mass density, $T$ the temperature and $\boldsymbol{u}$ the velocity of the flow. The most popular model for the collision term is the BGK approximation proposed by Bhatnagar, Gross and Krook [23] which states that the distribution function tends to the equilibrium distribution function in a characteristic time $\tau_{f}$ (often referred to as relaxation time):

$$
\left(\frac{\partial f(\boldsymbol{x}, t, \boldsymbol{\xi})}{\partial t}\right)_{\text {coll }}=-\frac{1}{\tau_{f}}\left(f(\boldsymbol{x}, t, \boldsymbol{\xi})-f^{\mathrm{eq}}(\boldsymbol{x}, t, \boldsymbol{\xi})\right) .
$$

The link from mesoscopic to macroscopic scale is made by integrating the distribution function over the velocity space. The mass density $\rho(\boldsymbol{x}, t)$, momentum density $\rho(\boldsymbol{x}, t) \boldsymbol{u}(\boldsymbol{x}, t)$ and total energy density $\rho(\boldsymbol{x}, t) E(\boldsymbol{x}, t)$, with $E(\boldsymbol{x}, t)$ the specific total energy, indeed corresponds to the $0^{\text {th }}$ order, $1^{\text {st }}$ order and $2^{\text {nd }}$ order moments respectively:

$$
\begin{gathered}
\rho(\boldsymbol{x}, t)=\int_{\mathfrak{R}^{3}} f(\boldsymbol{x}, t, \boldsymbol{\xi}) \mathrm{d} \boldsymbol{\xi}, \\
\rho(\boldsymbol{x}, t) \boldsymbol{u}(\boldsymbol{x}, t)=\int_{\mathfrak{R}^{3}} \boldsymbol{\xi} f(\boldsymbol{x}, t, \boldsymbol{\xi}) \mathrm{d} \boldsymbol{\xi}, \\
\text { and } \rho(\boldsymbol{x}, t) E(\boldsymbol{x}, t)=\frac{1}{2} \int_{\mathfrak{R}^{3}}|\boldsymbol{\xi}|^{2} f(\boldsymbol{x}, t, \boldsymbol{\xi}) \mathrm{d} \boldsymbol{\xi} .
\end{gathered}
$$

With $\tau_{f}=v / c^{2}$ where $v$ the kinematic viscosity and $c$ is the speed of sound, the NS equations for fluids with a Prandtl number of unity are recovered through a Chapman-Enskog expansion. For fluids with different Prandtl number, at least 
two relaxation times are required to correctly uncouple aerodynamic and thermodynamic relaxation processes [24].

In order to be solved, these equations need to be discretized not only in space and time like in standard NS solvers but also in the velocity space [25]. In the method, it is assumed that a particle covers exactly the distance $\Delta x$ between two adjacent lattice nodes during a time interval $\Delta t$, thus defining a Courant-Friedrichs-Lewy (CFL) number of unity. The discrete velocities are written $\boldsymbol{\xi}_{\alpha}$, the corresponding distribution functions $f_{\alpha}(\boldsymbol{x}, t)$ and the corresponding equilibrium distribution functions $f_{\alpha}^{\mathrm{eq}}(\boldsymbol{x}, t)$. Eqs. (1)-(3) therefore become, after integration over the time interval $\Delta t$, a set of 19 discrete equations where the advection term on the left-hand side simply reduces to a streaming operation for a Lagrangian approach:

$$
f_{\alpha}\left(\boldsymbol{x}+\boldsymbol{\xi}_{\alpha} \Delta t, t+\Delta t\right)-f_{\alpha}(\boldsymbol{x}, t)=-\frac{1}{\tau_{f}} \int_{0}^{\Delta t}\left(f_{\alpha}(\boldsymbol{x}, t)-f_{\alpha}^{\mathrm{eq}}(\boldsymbol{x}, t)\right) \mathrm{d} t
$$

Approximating the right-hand side of Eq. (7) with the trapezoidal rule and introducing the change of variable $g_{\alpha}(\boldsymbol{x}, t)=f_{\alpha}(\boldsymbol{x}, t)+\Delta t /\left(2 \tau_{f}\right)\left[f_{\alpha}(\boldsymbol{x}, t)-f_{\alpha}^{\mathrm{eq}}(\boldsymbol{x}, t)\right]$ yields the second-order lattice Boltzmann equation:

$$
g_{\alpha}\left(\boldsymbol{x}+\boldsymbol{\xi}_{\alpha} \Delta t, t+\Delta t\right)=g_{\alpha}(\boldsymbol{x}, t)-\frac{\Delta t}{\tau_{g}}\left(g_{\alpha}(\boldsymbol{x}, t)-g_{\alpha}^{\mathrm{eq}}(\boldsymbol{x}, t)\right),
$$

with $\tau_{g}=\tau_{f}+\Delta t / 2$ and $g_{\alpha}^{\mathrm{eq}}(\boldsymbol{x}, t)=f_{\alpha}^{\mathrm{eq}}(\boldsymbol{x}, t)$. This equation is solved in a two-step stream and collide algorithm that makes the efficiency of the method (local collision and exact advection):

$$
\begin{gathered}
\text { collision : } \hat{g_{\alpha}}(\boldsymbol{x}, t)=g_{\alpha}(\boldsymbol{x}, t)-\frac{\Delta t}{\tau_{g}}\left(g_{\alpha}(\boldsymbol{x}, t)-g_{\alpha}^{\mathrm{eq}}(\boldsymbol{x}, t)\right), \\
\text { streaming : } \quad g_{\alpha}\left(\boldsymbol{x}+\boldsymbol{\xi}_{\alpha} \Delta t, t+\Delta t\right)=\hat{g_{\alpha}}(\boldsymbol{x}, t) .
\end{gathered}
$$

The link with macroscopic quantities is now done in the discretized velocity space such that:

$$
\begin{gathered}
\rho(\boldsymbol{x}, t)=\sum_{\alpha} f_{\alpha}(\boldsymbol{x}, t) \\
\rho(\boldsymbol{x}, t) \boldsymbol{u}(\boldsymbol{x}, t)=\sum_{\alpha} \boldsymbol{\xi}_{\alpha} f_{\alpha}(\boldsymbol{x}, t) . \\
\text { and } \rho(\boldsymbol{x}, t) E(\boldsymbol{x}, t)=\frac{1}{2} \sum_{\alpha}\left|\boldsymbol{\xi}_{\alpha}\right|^{2} f_{\alpha}(\boldsymbol{x}, t) .
\end{gathered}
$$

Note that in order to recover the equilibrium moments with only a few discrete velocities, the equilibrium distribution functions $f_{\alpha}^{\mathrm{eq}}(\boldsymbol{x}, t)$ are expressed as a projection on the Hermite polynomials and a Gauss-Hermite quadrature is applied [26]. The expansion can then be truncated to a certain order depending on the lattice. For 3D applications, the ProLB solver is based on a 19-velocities discretization (D3Q19 lattice illustrated in Fig. 2). A third-order truncation of 
the equilibrium distribution functions is used and writes:

$$
f_{\alpha}^{\mathrm{eq}}(\boldsymbol{x}, t)=\rho \omega_{\alpha}\left[1+\frac{\xi_{\alpha} \cdot \boldsymbol{u}}{r T}+\frac{\left(\xi_{\alpha} \cdot \boldsymbol{u}\right)^{2}}{2 r^{2} T^{2}}-\frac{|\boldsymbol{u}|^{2}}{2 r T}+\frac{\xi_{\alpha} \cdot \boldsymbol{u}}{6 r T}\left(\frac{\left(\xi_{\alpha} \cdot \boldsymbol{u}\right)^{2}}{r^{2} T^{2}}-\frac{3|\boldsymbol{u}|^{2}}{r T}\right)\right],
$$

with $r=k_{B} / m$ the gas constant and $\omega_{\alpha}$ the weighed coefficients associated with the Hermite polynomials. A second-order truncation should be sufficient in theory for this scheme but using the third-order truncation brings more precision and stability. This discretization allows the $0^{\text {th }}, 1^{\text {st }}$ and $2^{\text {nd }}$ order moments to be recovered and introduces an $\mathrm{O}\left(M^{3}\right)$ error in the $3^{\text {rd }}$ order moment, $M$ being the Mach number. As a consequence, an $\mathrm{O}\left(M^{3}\right)$ error is introduced in the momentum conservation equation through the shear stress and restricts the use of this lattice to athermal and quasi-incompressible flows [12]. For higher Mach number values, one solution is to take more discrete velocities to allow for a higher-order truncation.

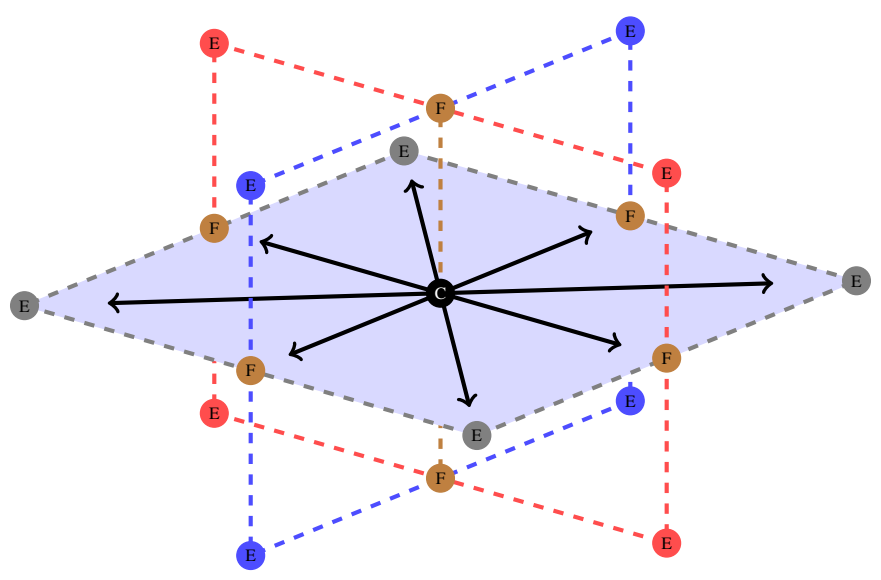

Fig. 2 Representation of the D3Q19 lattice. The black node (C) points to the center of the lattice, the brown nodes (F) point to the faces of the neighboring cubes, and the grey (E), blue (E) and red nodes (E) point to the edges of the neighboring cubes. For clarity, only the velocities in the horizontal plane $(\square)$ are displayed. Reprinted from the paper of Hainaut et al. [27].

Some specificities of the ProLB solver are now detailed. For better robustness and accuracy, a variant of the BGK collision term based on two relaxation times is implemented. This so-called Dual Relaxation Time (DRT) method consists of over-relaxing the distributions towards equilibrium while preserving mass and momentum conservation, and reconstructing the non-equilibrium part through a second-order regularization process [3]. A turbulence model is also adopted to avoid the prohibitive cost of Direct Numerical Simulations (DNS). The Shear-improved Smagorinsky model of Lévêque et al. [28] is chosen here. Finally, for turbofan applications, a specific treatment is needed to deal with the rotor motion. It is achieved in the ProLB solver with an Arbitrary Lagrangian-Eulerian (ALE) approach [29]. 


\section{B. Setup of the ANCF case}

\section{Geometry and Operating Conditions}

The 2008 geometry of the ANCF with upgraded fan blades and Inlet Control Device (ICD) is considered in this paper. Among the various available configurations, the one with the most complete measurements, which corresponds to the 14-vane stator spaced from the rotor by one rotor chord, is chosen. The corresponding geometry is shown in Fig. $3($ a). Note that the upper lip of the casing has been extended up to the exhaust exit plane in the simulations for simplification. In addition, the ICD is not included and the rotor tip gap is neglected. The overall fluid domain in the simulations is extended up to 12 rotor diameters in all directions.

Nominal operating conditions are investigated, corresponding to a rotation speed of $1800 \mathrm{rpm}$, because it is the only regime where radial flow profiles inside the duct have been measured. The experimental data on this configuration are acquired during several days so that the ambient pressure and temperature can change. For the simulations, averaged conditions given by an ambient pressure of 99,330 Pa and an ambient temperature of $290 \mathrm{~K}$ are chosen.

Note that for further aerodynamic evaluations, an additional configuration corresponding to the RANS results provided in the database (i.e. rotation speed of $2000 \mathrm{rpm}$ and 14 stator vanes spaced from the rotor by $0.27 c_{R}$ ) will also be considered in this paper.

\section{Boundary Conditions}

An outlet pressure condition is set at all external fluid boundaries, except for the one that faces the nacelle inlet for which an inlet velocity condition is applied. The inlet velocity applied is very low (corresponding to a Mach number of 0.01) but still allows for the streamlines to be generated upstream of the nacelle. Absorbing regions are added on these external boundaries to avoid spurious reflections inside the fluid domain. Finally, wall boundary conditions (with the use of a wall function) are applied on all solid surfaces.

\section{Resolution Domains}

Even if there is no meshing step in LBM solvers, resolution domains are defined in order to have the finest cells only where required. As already mentioned, the lattice imposes the space discretization such that only a stretching by a factor of 2 in all space directions is possible at the transition between two successive resolution domains. This means that, if $\Delta x_{\min }$ is the smallest cell size and $N$ is the number of resolutions domains, the cell size in the resolution domain $n$, with $n=1$ for the finest domain and $n=N$ for the coarsest one, is equal to $2^{n-1} \Delta x_{\min }$. Note that, the CFL in LBM simulations being intrinsically of unity, the distribution functions are updated only every $2^{n-1}$ iterations for the resolution domain $n$. In the present simulations, 10 resolution domains are defined in total and are shown in Fig. 3(b)-(c). The first resolution domain with cell size $\Delta x_{\min }=0.75 \mathrm{~mm}$ encompasses the rotor blades with at least 12 layers. The second resolution domain comprises the whole rotor-stator system. All hub and shroud walls are enveloped 
by the third and fourth resolution domains. The fifth resolution domain, where the cell size is of $12 \mathrm{~mm}$, includes the far-field microphones. Acoustic waves up to 6BPF are discretized with at least 10 points per wavelength in this volume in order to provide direct acoustic evaluation. This drastically increases the total number of nodes but their relative cost compared to the one of the finest points is low because they are updated only one every $2^{4}$ iterations. Successive resolution domains are then added up to the external boundaries. The coarsest cell size is about $0.4 \mathrm{~m}$, which is more than half the wavelength associated to the BPF.

For mesh convergence assessment, three simulations based on this description are achieved at nominal operating condition: a coarse simulation $\left(\Delta x_{\min }=3 \mathrm{~mm}\right)$ where both the first and the second resolution domains are deactivated, a medium simulation $\left(\Delta x_{\min }=1.5 \mathrm{~mm}\right)$ where only the first resolution domain is deactivated and a fine simulation $\left(\Delta x_{\min }=0.75 \mathrm{~mm}\right)$ that includes all the resolution domains. The study of the mesh convergence is therefore restricted to the rotor-stator region. This choice is made because the propagation of acoustic waves outside of this region is not expected to be altered by the discretization as soon as there is at least 10 points per wavelength. The effect of refining the turbulent structures of the rotor blade wakes (which cause the acoustic sources on the stator vanes when interacting with them) is included in the coarse/medium comparison, as well as the capability of the wall law to correctly reproduce the physics for a given non-dimensionalized first cell size $y^{+}$. Because the $y^{+}$values are expected to be more important over the rotor blades, the latter effect is further analyzed by the medium/fine comparison. This setup lead to maximum values of $y^{+}$on the rotor blades of about 900,500 and 250 for the coarse, medium and fine simulations respectively. Note that an additional fine simulation is performed at $2000 \mathrm{rpm}$ and with adjusted rotor-stator space for comparison with the available RANS results. All the simulated configurations are summed up in Table 1 .

Table 1 Performed simulations. RD stands for Resolution Domain.

\begin{tabular}{ccccc} 
Label & First active RD & Rotation speed & Stator vanes & Rotor-stator space \\
\hline Coarse $/ 1800$ & 3 & $1800 \mathrm{rpm}$ & 14 & $c_{R}$ \\
Medium $/ 1800$ & 2 & $1800 \mathrm{rpm}$ & 14 & $c_{R}$ \\
Fine $/ 1800$ & 1 & $1800 \mathrm{rpm}$ & 14 & $c_{R}$ \\
Fine $/ 2000$ & 1 & $2000 \mathrm{rpm}$ & 14 & $0.27 c_{R}$ \\
\hline
\end{tabular}

\section{Numerical Parameters}

The total number of nodes is about 260 millions, 370 millions and 422 millions for the coarse, medium and fine simulations at nominal operating point respectively. For LBM solvers, it is more representative to speak about Fine Equivalent (FE) nodes, which represents the number of nodes that are effectively updated at each solver iteration. The total cost of a simulation therefore scales with this number times the number of smallest time iterations. The coarse, medium and fine meshes have 110 millions, 170 millions and 141 millions FE nodes respectively. However, it should be kept in mind that the time step decreases when activating the finest resolution domains, thus increasing the number 


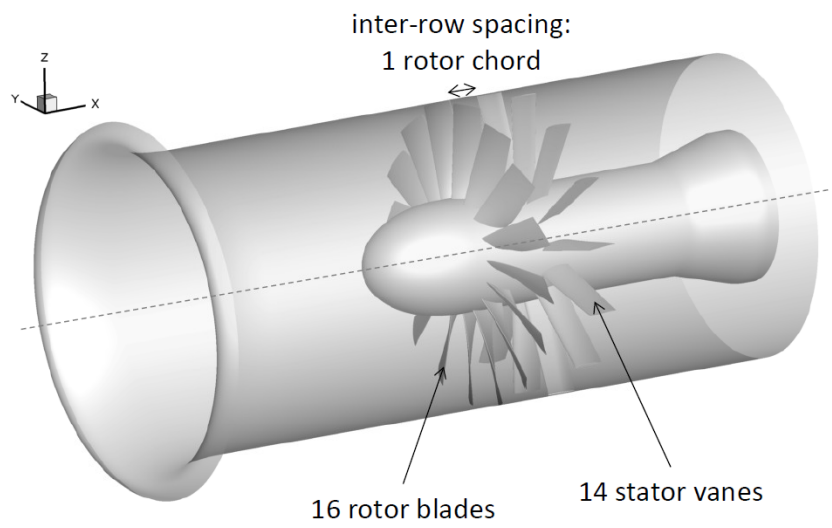

(a) Geometry of the chosen configuration (without the extended upper lip).

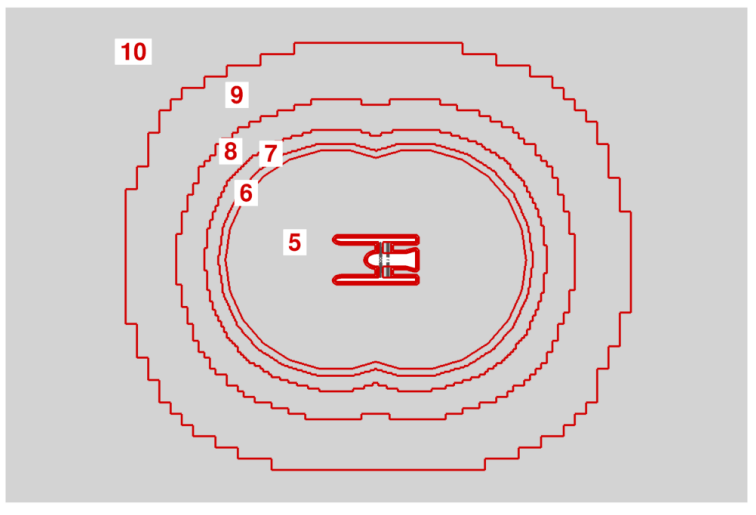

(b) Global view of the resolution domains.

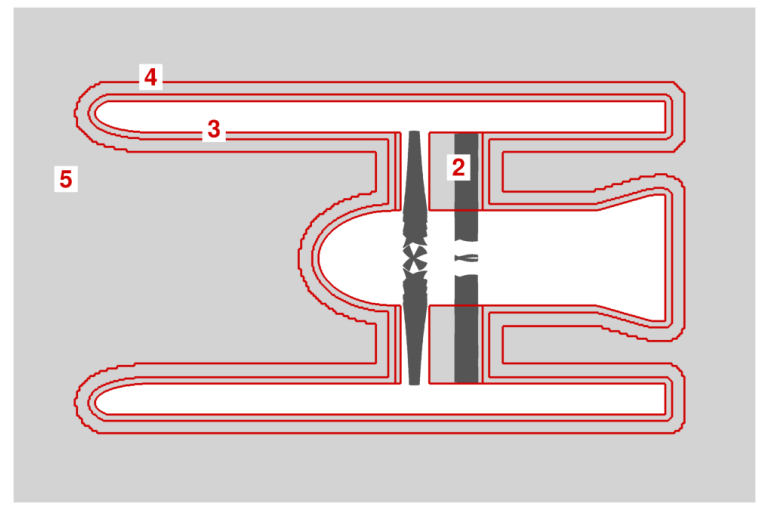

(c) Zoomed view of the resolution domains.

Fig. 3 Geometry of the chosen configuration and definition of the resolution domains (the first resolution domain corresponding to an offset around rotor blades is not shown). 
of iterations needed to simulate a given physical time. For the fine simulation, the time step is of $1.28 \mu \mathrm{s}$, which corresponds to 26,112 iterations per rotation or 1632 iterations per blade passage. The time step is twice larger and the number of iterations per rotation twice lower for the medium case. For the coarse simulation, there is an additional factor of two. All simulations are run for an equivalent physical time corresponding to 20 complete rotor rotations. This is sufficient to converge the unsteady phenomena and to provide reasonable statistics for noise predictions. For the coarse simulation, 448 cores are used and one rotor rotation is computed in approximately one hour (total computational cost of 9000 hours). As for the medium and fine simulations, they are run over 896 cores and they require 3.6 and 5 hours per rotor rotation respectively (total cost of 65,000 and 90,000 hours). Note that the ProLB solver has not been used at its optimal CPU load factor (priority put on retrieval time) as evidenced by the small difference in total cost between the medium and fine cases (observed factor of 1.38 instead of a theoretical factor of 1.66 with perfect CPU load balance).

The additional fine simulation performed at $2000 \mathrm{rpm}$ and with adjusted rotor-stator space for comparison with the RANS results totalizes 438 millions total nodes and 146 millions FE nodes. The small difference with the nominal operating point fine simulation comes from small differences in the mesh cell size (less than $0.01 \mathrm{~mm}$ ) that allow keeping an integer number of iterations per rotation (for post-processing purpose). 23,808 iterations per rotation are now used and the time step is reduced to $1.26 \mu$ s. Contrary to the other simulations, only aerodynamic results are expected from this additional simulation so that only 6 rotor rotations are achieved (it will be shown later that this is sufficient for aerodynamic convergence). All the above parameters are summarized in Table 2 .

Table 2 Simulation characteristics.

\begin{tabular}{cccccccc} 
Label & $\Delta x_{\min }$ & $y_{\max }^{+}$ & Total nodes & FE nodes & $\Delta t_{\min }$ & Time steps & CPU Time \\
\hline Coarse $/ 1800$ & $3 \mathrm{~mm}$ & 900 & $260 \times 10^{6}$ & $110 \times 10^{6}$ & $5.11 \times 10^{-6} \mathrm{~s}$ & $20 \times 6528$ & $9000 \mathrm{~h}$ \\
Medium $/ 1800$ & $1.5 \mathrm{~mm}$ & 500 & $370 \times 10^{6}$ & $170 \times 10^{6}$ & $2.55 \times 10^{-6} \mathrm{~s}$ & $20 \times 13,056$ & $65,000 \mathrm{~h}$ \\
Fine $/ 1800$ & $0.75 \mathrm{~mm}$ & 250 & $422 \times 10^{6}$ & $141 \times 10^{6}$ & $1.28 \times 10^{-6} \mathrm{~s}$ & $20 \times 26,112$ & $90,000 \mathrm{~h}$ \\
Fine $/ 2000$ & $0.75 \mathrm{~mm}$ & 250 & $438 \times 10^{6}$ & $146 \times 10^{6}$ & $1.26 \times 10^{-6} \mathrm{~s}$ & $6 \times 23,808$ & $23,000 \mathrm{~h}$ \\
\hline
\end{tabular}

\section{Aerodynamic Results}

Throughout the paper, the focus is essentially put on the 14-vane configuration spaced by one rotor chord from the rotor and at nominal operating conditions (the most instrumented case). Consequently, if not stated otherwise, the simulation results that will be shown will correspond to this configuration. The results of the 2000 rpm case with reduced rotor-stator spacing will only be used for comparison with the database RANS results and will help us to understand the aerodynamic simulations/measurements comparison of the nominal case. 


\section{A. Basic Flow Patterns}

Before going into quantitative results, the basic flow patterns obtained from the fine simulation are first shown in Fig. 4 for qualitative visualizations. In Fig. 4 a), a radial slice at $r=0.4 \mathrm{~m}$ colored by the axial velocity (black and white map) and an isosurface of Q-criterion at $5 \times 10^{5} \mathrm{~s}^{-2}$ colored by the vorticity magnitude (colored map) are shown. They highlight the wakes created by the rotor blades, their advection in the interstage and finally their impact with the stator vanes. It should be noted that the turbulent structures seem to be correctly advected through the mobile/fix interface located in between the rotor and the stator. The acceleration of the flow past the suction side of rotor blades and the stator vane wakes are also evidenced by the axial velocity contours. In Fig. 4(b), an isosurface of Q-criterion colored by the vorticity magnitude (colored map) is shown again but at a different value $\left(5 \times 10^{6} \mathrm{~s}^{-2}\right)$ to highlight the turbulent structures downstream of the nacelle. An horizontal slice at $\mathrm{z}=0 \mathrm{~m}$ colored by density gradient magnitude is also plotted (black and white map) and shows the acoustic waves radiated by the turbofan correctly captured by the LBM simulation. These plots give an illustration of the philosophy of the approach adopted here, which is particularly suited for LBM simulations: in a single computation, both the unsteady aerodynamics and the generation and propagation of acoustic sources up to the far-field microphones (where the noise is generally measured) are predicted. This direct approach can of course also be carried out with NS solvers but the cost to accurately propagate acoustic waves over long distances limits in practice its application.

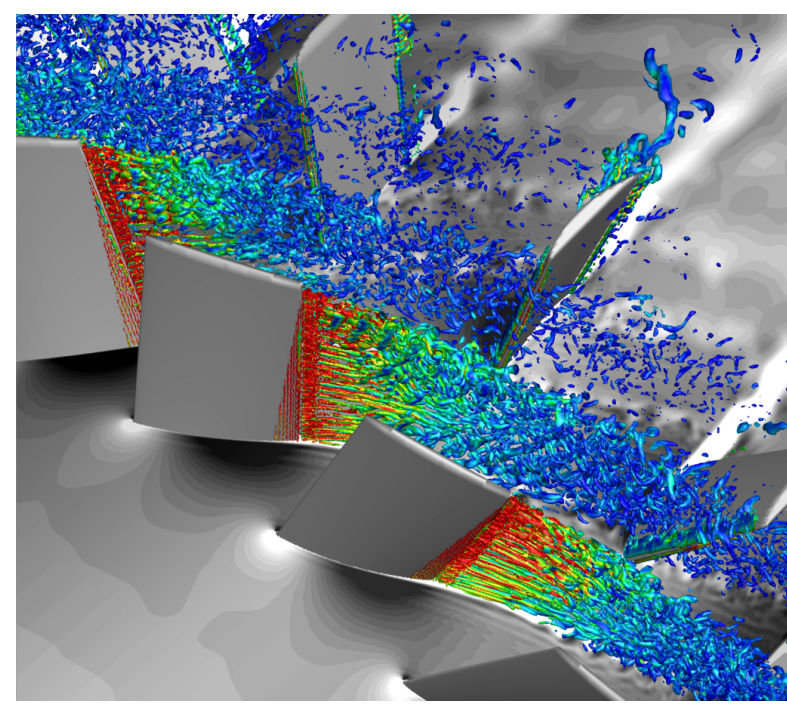

(a) Radial slice at $\mathrm{r}=0.4 \mathrm{~m}$ colored by axial velocity (in black and white) and isosurface of Q-criterion at $5 \times 10^{5} \mathrm{~s}^{-2}$ colored by vorticity magnitude (in colors).

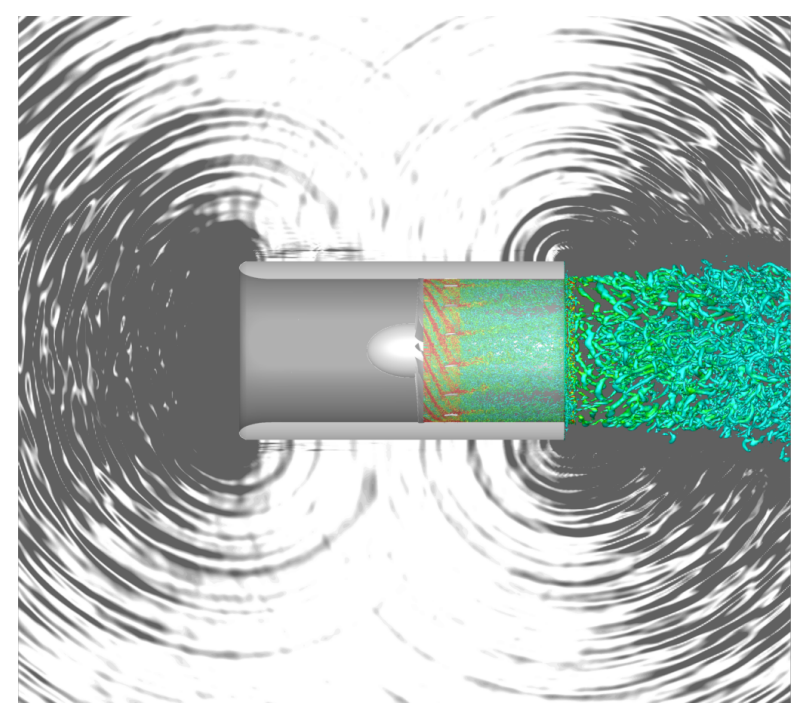

(b) Horizontal slice at $\mathrm{z}=0 \mathrm{~m}$ colored by density gradient magnitude (in black and white) and isosurface of Q-criterion at $5 \times 10^{6} \mathrm{~s}^{-2}$ colored by vorticity magnitude (in colors).

Fig. 4 Instantaneous basic flow patterns obtained from the fine simulation. 


\section{B. Fan Performance}

The fan performance is analyzed as it is of first importance for turbomachines. The evolutions of the mass flow rate of air and of the total pressure ratio during the simulations are shown in Fig. 5. Experimental values are given for the comparison. Note that the experimental total pressure ratio has been deduced from measurements of upstream and downstream total pressure profiles. Both profiles have not been measured simultaneously and it appears that the ambient pressure has changed by $400 \mathrm{~Pa}$ in the meantime. In order not to alter the pressure ratio, it is computed here considering the same ambient pressure $(99,330 \mathrm{~Pa})$ for both measurements. This correction is not negligible for such low pressure ratio (decrease by 0.03 approximately). For all simulations, the convergence of mass flow rate and total pressure ratio is obtained after about 5 rotations (starting from an initial fluid at rest). The mesh convergence for performance prediction is reached with very close levels obtained for the medium and the fine simulations. However, even for the fine simulation, the discrepancies with the measurements are high for the mass flow rate which is lower by $8.5 \%$. The total pressure ratio is lower by only $0.4 \%$ but this number is not significant given the low absolute pressure ratio value of the present turbofan. By focusing on the pressure jump error, defined by:

$$
\eta_{\Delta \Pi}=\frac{\Pi_{\mathrm{LBM}}-\Pi_{\text {Measurements }}}{\Pi_{\text {Measurements }}-1},
$$

where $\Pi_{L B M}$ and $\Pi_{\text {Measurements }}$ denote the LBM and experimental total pressure ratio respectively, the underestimation becomes $22.3 \%$. These differences are much higher than the tolerance generally admitted for turbomachinery applications (around 1\%). As already noticed in Sec. I. a similar underestimation of the mass flow rate was also obtained in the previous studies performed with the PowerFLOW solver [13, 15]. More investigations on that aspect are done in Sec.IV.D

\section{Radial Flow Profiles}

Mean flow profiles are now compared with measurements from radial traverses of two-component hotfilm and pressure probes located one chord upstream of the rotor and one chord downstream of the rotor. For the upstream traverse, the hotfilm gives access to axial and radial velocity components while for the downstream one, it gives access to axial and circumferential components. The experimental data is averaged over 500 rotor rotations. The simulated radial flow profiles are obtained by performing circumferential averages of the time-averaged flow at successive radial positions. The flow is time-averaged over the last rotor rotation and it has been checked that this average does not change during the last three rotations.

The results for the inlet traverse in terms of total pressure, static pressure, flow velocity and flow angle are given in Fig. 6. The relative total pressure in the simulations is around $0 \mathrm{~Pa}$, which means that the total pressure is correctly conserved from the far-field to the duct entrance. This is not the case in the experiments and the pressure loss of about 


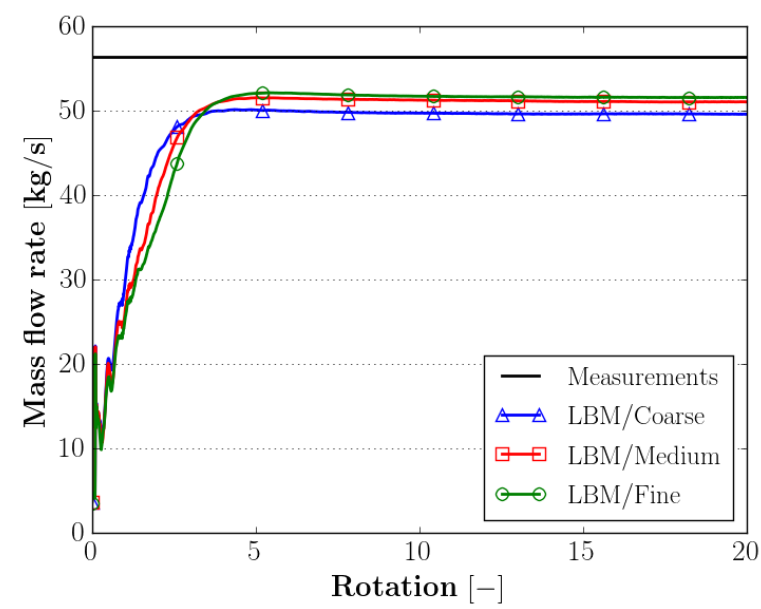

(a) Mass flow rate.

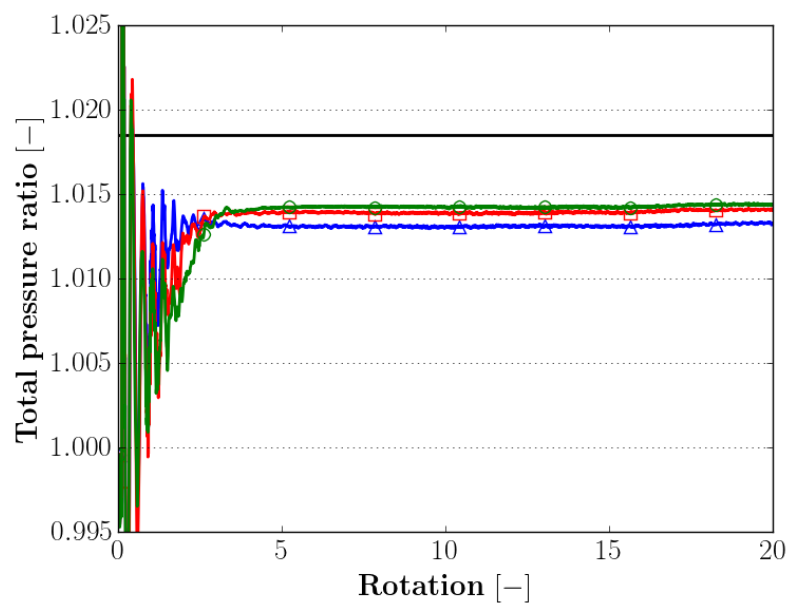

(b) Total pressure ratio.

Fig. 5 Evolution of fan performance values during the simulations: effect of mesh refinement and comparison with experiments.

$80 \mathrm{~Pa}$ can be attributed to the ICD [21], not represented in the simulations. The static pressure and velocity profiles are the only ones significantly affected by the mesh resolution, even if the results obtained with the medium and fine meshes are very close. When refining, the flow velocity increases and becomes very close to the measured profile. In the same time, the static pressure decreases and is getting closer to the experimental curve. The shapes of the static pressure profiles are similar to the experimental one but even for the fine simulation, the absolute values differ significantly. The static pressure is overestimated by about $400 \mathrm{~Pa}$ (fine simulation), so this gap cannot be attributed only to the pressure loss caused by the ICD (around $80 \mathrm{~Pa}$ ). The radial flow angle comparison is more surprising. The simulated curves present a classical behavior with an angle close to 0 degree at the tip where the duct wall is parallel to the duct axis and with a small non-zero angle at lower radius due to the curvature of the hub. The experimental curve does not have these expected characteristics. After discussions with NASA, it appeared that hotfilm calibration issues were encountered during the experiments making the absolute values of the measured angles not reliable. The experimental curve is therefore arbitrarily shifted by 5 degrees (dashed line) to provide at least a relative comparison. Even when shifted, the profiles do not compare very well. It is not easy to interpret these data so that no conclusion will be drawn from this plot. Nevertheless, the radial flow angles are quite low (below 10 degrees) so that they should not significantly impact the fan performance. What might be surprising at this stage is that the mass flow rate is underestimated in the fine simulation while 1) the flow velocity matches the experimental profile and 2) the static pressure (and therefore the density) is higher than the measured profile. These observations tend to indicate some uncertainty in the present experimental data.

Basic thermodynamic analysis is first suggested here to check this point. By using the isentropic flow relationships 


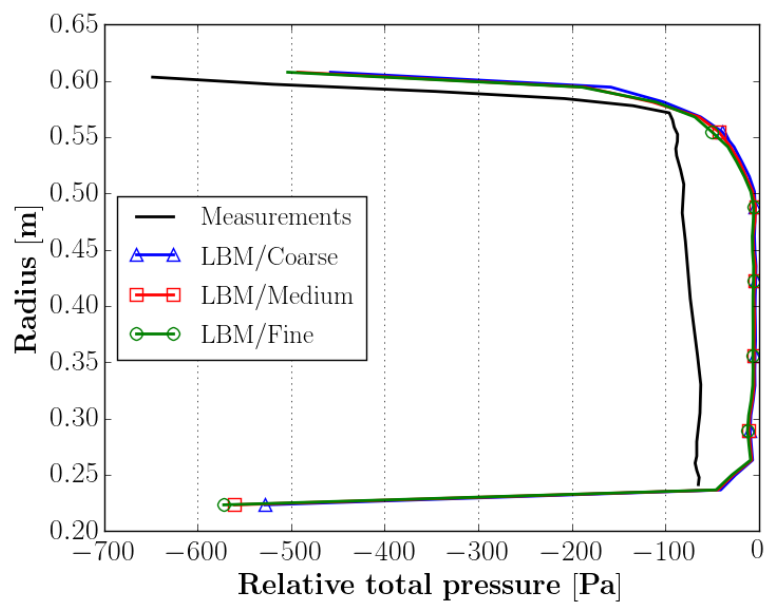

(a) Relative total pressure.

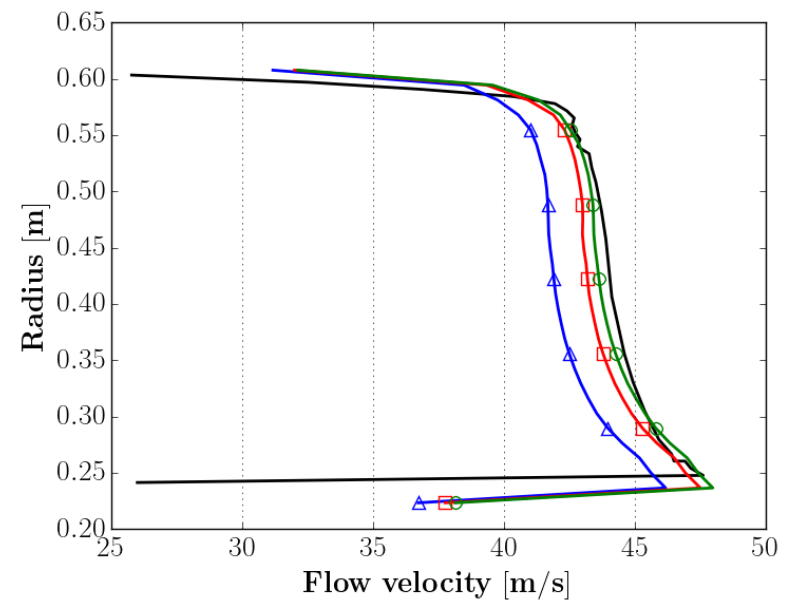

(c) Flow velocity.

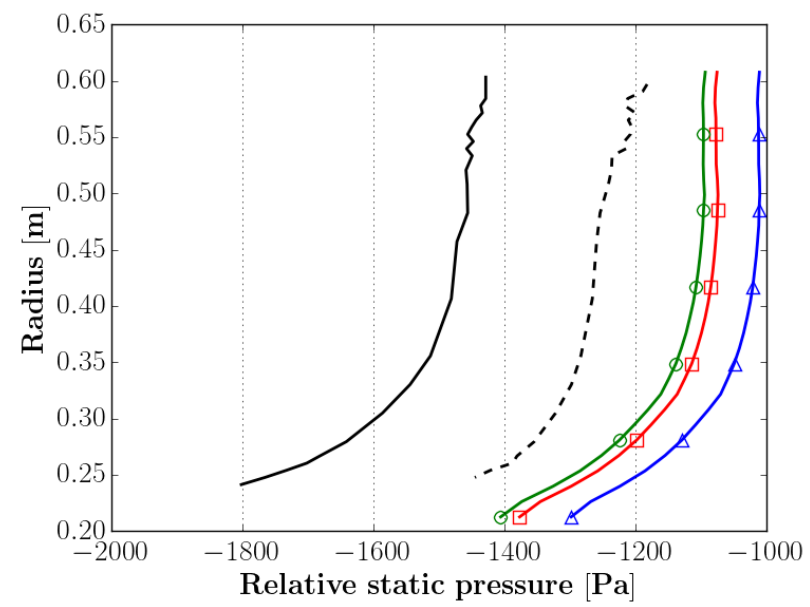

(b) Relative static pressure (the dashed line shows the corrected experimental curve)

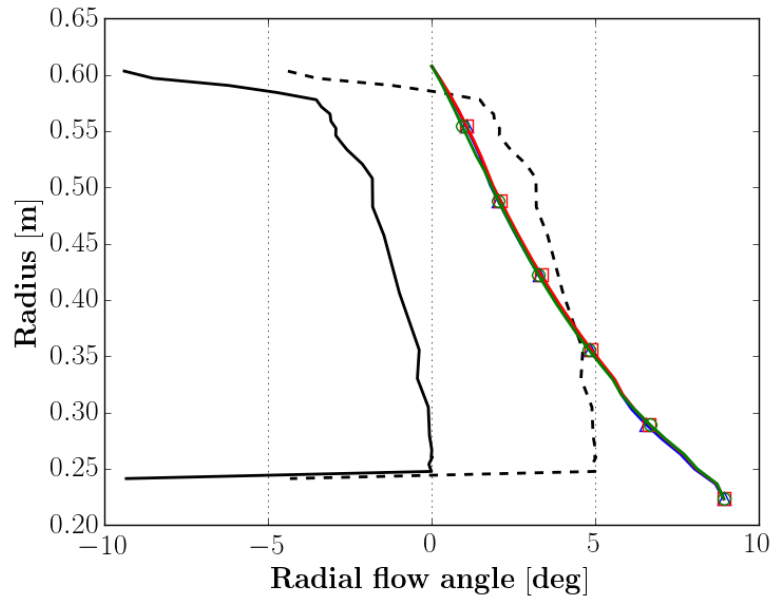

(d) Radial flow angle (the dashed line shows the shifted experimental curve).

Fig. 6 Mean radial flow profiles extracted one chord upstream of the rotor: effect of mesh refinement and comparison with experiments. 
and the ideal gas equation of state, the following relations can be written:

$$
\begin{gathered}
\frac{p_{T}}{p}=\left(1+\frac{\gamma-1}{2} M^{2}\right)^{\frac{\gamma}{\gamma-1}}, \\
M=\frac{u}{c}, \\
\text { and } c=\sqrt{\gamma R T} .
\end{gathered}
$$

Conventional notations are used: $p_{T}$ is the total pressure, $p$ the static pressure, $M$ the Mach number, $u$ the velocity, $c$ the speed of sound and $T$ the temperature of the flow while $\gamma$ and $R$ represent the heat capacity ratio and the specific gas constant of air respectively. The Mach number profile, sound speed profile and static temperature profile can therefore be successively computed from the experimental total pressure, static pressure and flow velocity curves. These operations result in a temperature profile around $240 \mathrm{~K}$, which is not realistic. Indeed, the static temperature inside the duct can be otherwise estimated using the total ambient temperature $T_{T}$ (which is conserved) and the expected Mach number upstream of the fan (0.15) with the following isentropic relationship:

$$
\frac{T_{T}}{T}=\left(1+\frac{\gamma-1}{2} M^{2}\right)
$$

The resulting estimate is of $288.7 \mathrm{~K}$, showing that the temperature varies only slowly at such low Mach numbers (ambient value of $290 \mathrm{~K}$ ). This deviation highlights the uncertainty of the absolute values of some variables in the experimental mean flow data. This is a result of the intent that experimental mean flow data (contrary to data related to acoustics) was not calibrated with absolute levels as an objective with the encountered varying ambient conditions (in terms of pressure and temperature). In order to further illustrate this point, the experimental static pressure profile is re-computed from the experimental total pressure and velocity profiles using a static temperature of $288.7 \mathrm{~K}$ and Eqs. (16), (17) and (18). The result is added in Fig. 6(b) in dashed line. The difference with the simulated curves is greatly reduced and now lies between 100 and $200 \mathrm{~Pa}$ (with about $80 \mathrm{~Pa}$ still to be attributed to the absence of ICD in the simulations).

The results for the downstream traverse are not shown to keep conciseness but similar difficulties to perform absolute comparisons are encountered. Nevertheless, the predicted radial shapes compare well with the experimental ones, especially with the fine simulation, except for the static pressure where a deviation is seen close to the hub.

\section{Comparison with RANS results}

The difficulties explained above to get the correct absolute values of the measured profiles prevent the complete validation of the LBM aerodynamic results. The capabilities of the isothermal LBM to correctly predict the aerodynamic features are therefore futher evaluated by comparing the results with the RANS solution provided in the database. RANS simulations are known to be particularly suited for mean flow predictions so that the available RANS results will 
be considered as a reference for the present LBM simulations. The configuration at $2000 \mathrm{rpm}$ with the stator spaced from the rotor by $0.27 c_{R}$ has therefore also been computed with the ProLB solver. Tackling such a configuration is straightforward since geometries are handle by immersed boundary conditions and the stator always lies in the same resolution domain. Only a fine simulation has been done as the effect of mesh convergence has already been shown in Sec.IV.C

Five domains are defined in the RANS simulation and are shown in Fig.7 7 the inlet, the rotor, the stator, the nozzle and the exit free-stream. The rotor and stator parts are simulated using the 3D RANS solver SWIFT [30] while the other parts are computed using the axisymmetric RANS solver AVCS [31]. The solutions at the inlet and outlet interfaces of the rotor and stator domains were coupled through an iterative process involving circumferentially-averaged quantities (similar to a mixing plane strategy). As for the nozzle and exit free stream solutions, they were coupled to obtain the free-shear layer solution at the outer edge of the nozzle exit jet.

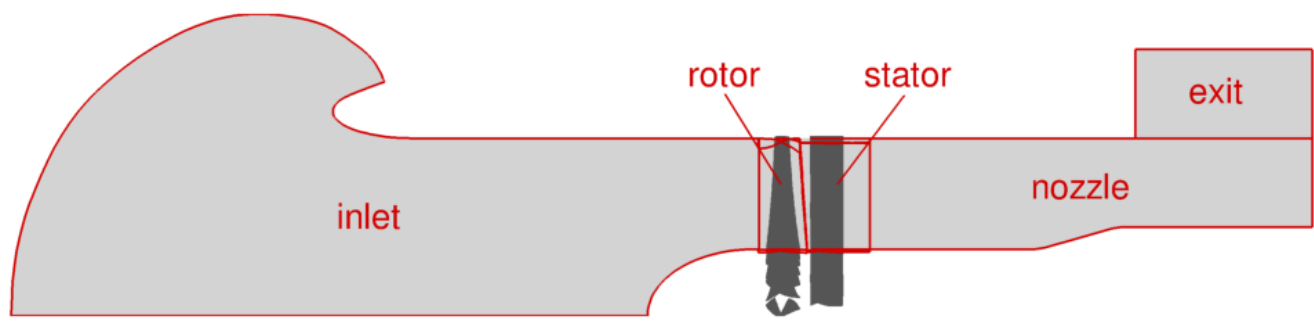

Fig. 7 Domains of the RANS simulation provided with the ANCF database. The red curves show the limits between the domains.

In this section, the RANS and LBM results are compared on the 2000 rpm case with reduced rotor-stator spacing but the LBM results at $1800 \mathrm{rpm}$ with one chord spacing between the rotor and the stator are also shown in order to outline the effects of the regime (rotor-stator spacing effects are expected to be less important). The comparison of fan performance prediction is first shown in Fig. 8 . The agreement between the RANS and LBM performance values is very good with a mass flow rate lower by $1.8 \%$ and a total pressure ratio lower by $0.1 \%$ in the LBM simulation (5.3\% by computing the error with respect to the reference value of 1 ). These results are much more promising than the previous comparisons between the LBM results and the measurements at nominal operating point.

The comparison of radial flow profiles is now performed one chord upstream of the rotor as done in Sec. IV.C The results are given in Fig. 9 and shows an excellent agreement between the RANS and LBM results at $2000 \mathrm{rpm}$. The only noticeable differences are seen on the static pressure with a maximum discrepancy of about $50 \mathrm{~Pa}$ and on the radial flow angle with a maximum deviation of 6 degrees close to the hub. It can also be noticed that the rotation speed essentially modifies the flow velocity and static pressure at this location since the relative total pressure remains around zero (total pressure is conserved) and the radial flow angle is mainly due to the hub curvature. When increasing the rotation speed, the flow velocity and static pressure are logically increased and decreased respectively. 


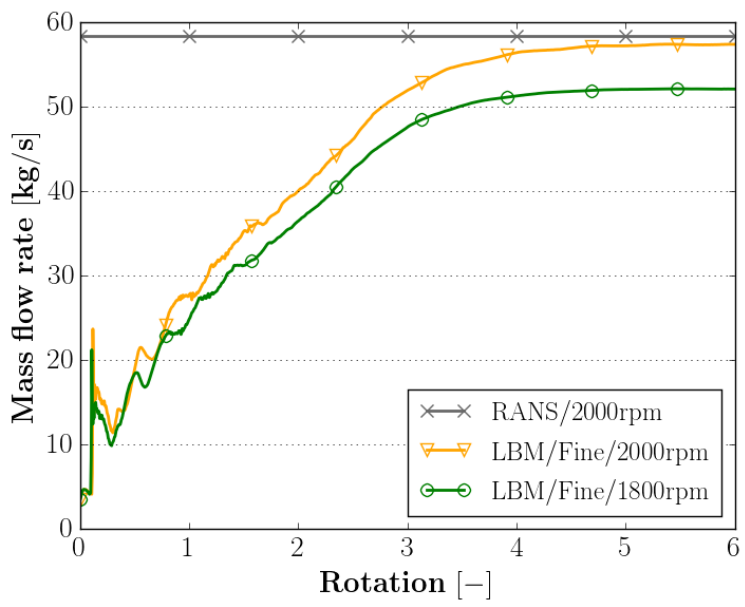

(a) Mass flow rate.

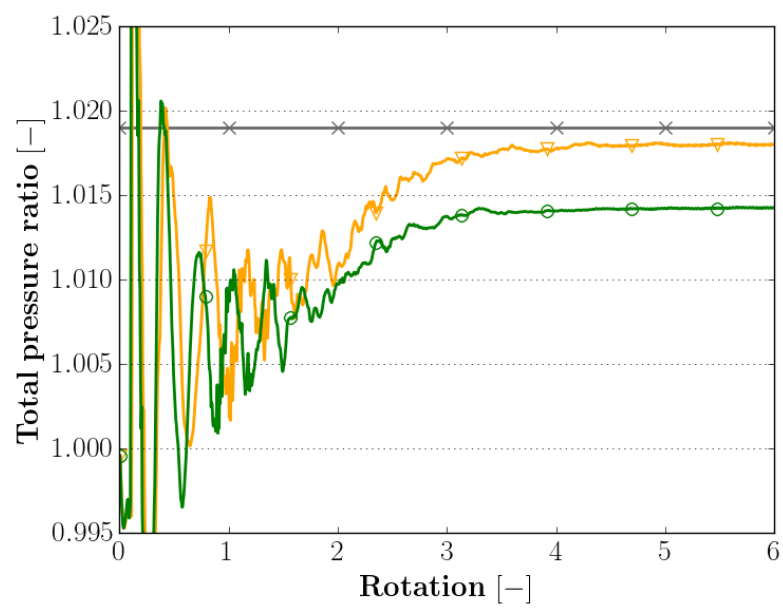

(b) Total pressure ratio.

Fig. 8 Evolution of fan performance values during the simulations: comparison with RANS results.

The results located one chord downstream of the rotor are not shown here, contrary to what was done in Sec. IV.C, because of the presence of the stator vanes at this location for this configuration with reduced interstage. The aft evaluation is therefore done further downstream, at approximately one chord from the stator (or about four chords from the rotor). The results, including again the ones at $1800 \mathrm{rpm}$ with one chord spacing between the rotor and stator for comparison, are shown in Fig. 10. The agreement between the RANS and LBM results is also very good at this location. The maximum differences are seen on the circumferential flow angle profile with a higher deviation of 4 degrees predicted by the LBM close to the hub. Increasing the rotation speed here affects all the variables by increasing the flow velocity, total pressure and static pressure and slightly decreasing the azimuthal flow angle. The comparisons shown in Figs. 8, 9 and 10 validate the isothermal LBM aerodynamic results in an absolute sense and therefore seem to confirm the uncertainty of the experimental data mentioned in Sec. IV.C

\section{Acoustic Results}

This section is devoted to the evaluation of the acoustic results of the present isothermal LBM simulations. Only the results of the 14-vane configuration spaced by one rotor chord from the rotor and at nominal operating conditions are depicted in the following.

\section{A. Broadband Noise}

\section{Direct Far-Field Pressure Spectra}

The broadband far-field acoustic results are first investigated. In the experiments, 30 microphones are located in the far-field: 15 microphones on a forward arc with a radius of about $3.66 \mathrm{~m}$ from the inlet plane and 15 microphones on a 


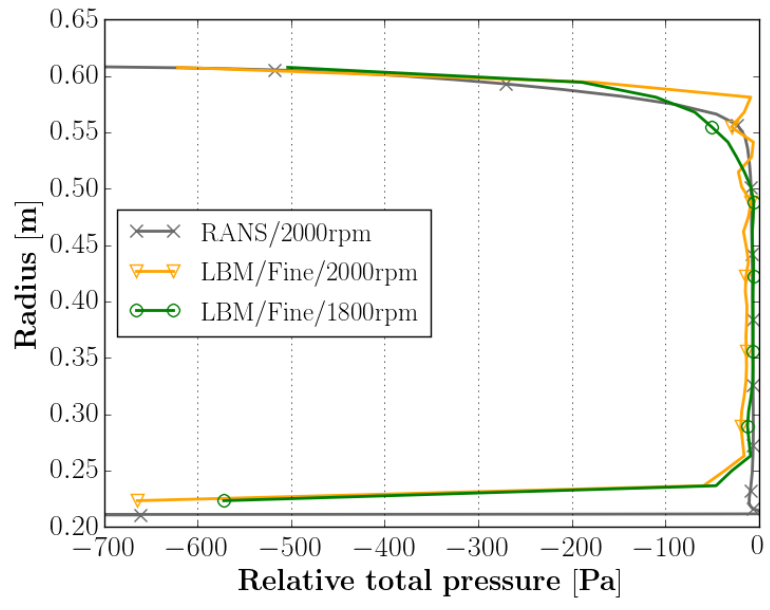

(a) Relative total pressure.

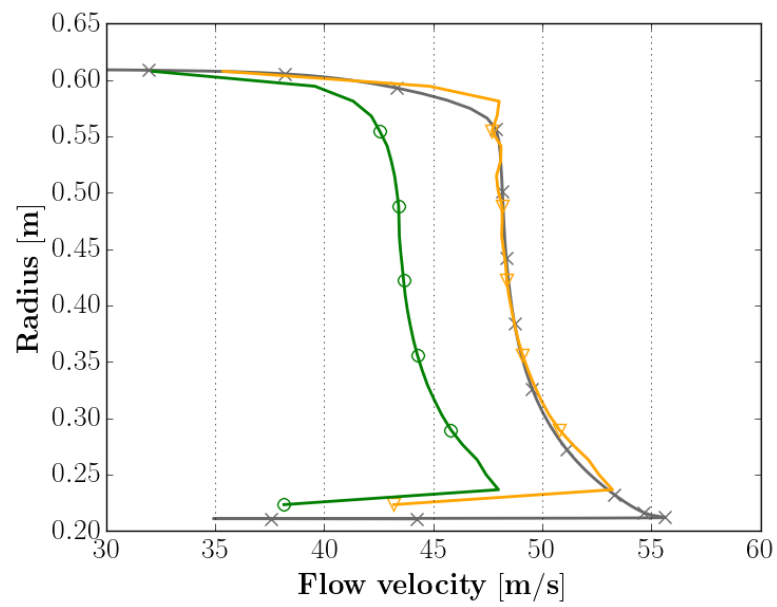

(c) Flow velocity.

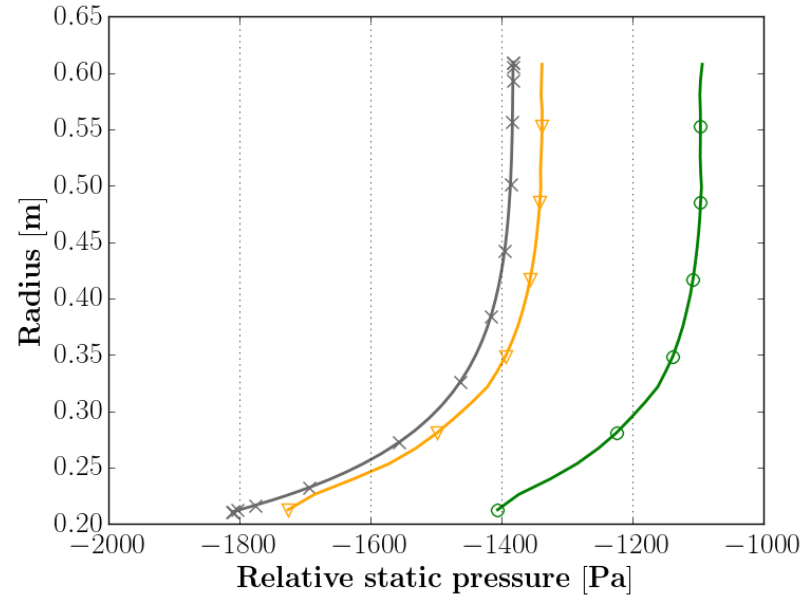

(b) Relative static pressure.

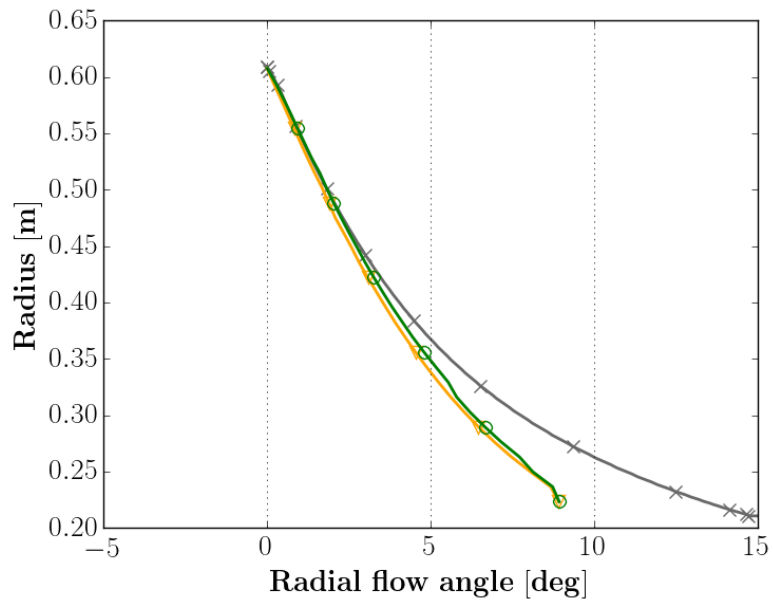

(d) Radial flow angle.

Fig. 9 Mean radial flow profiles extracted one chord upstream of the rotor: comparison with RANS results. 


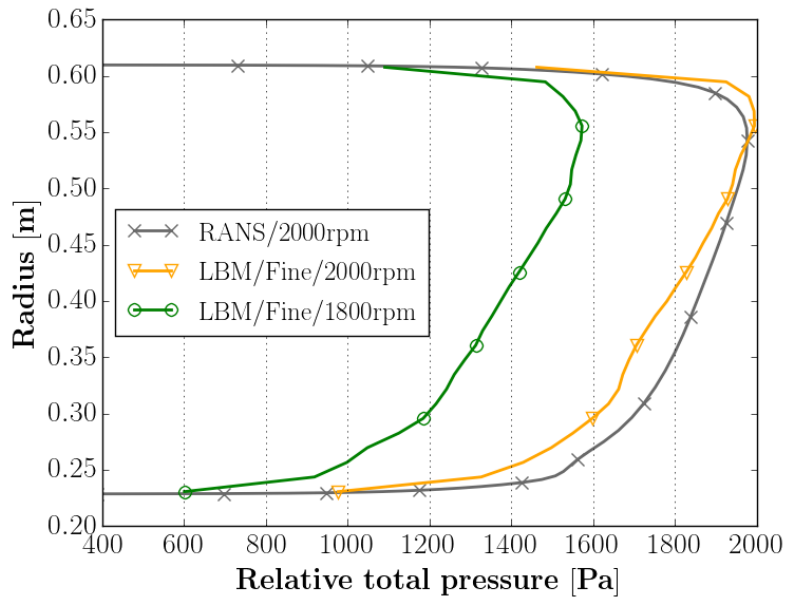

(a) Relative total pressure.

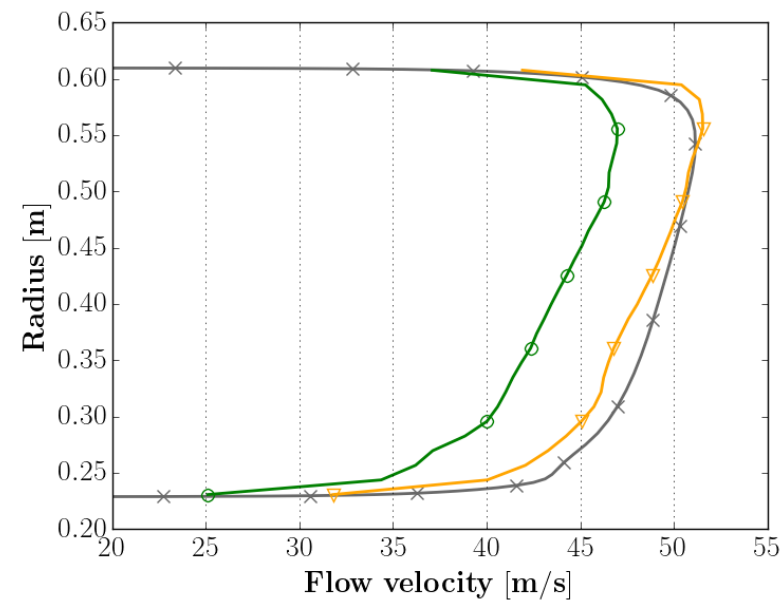

(c) Flow velocity.

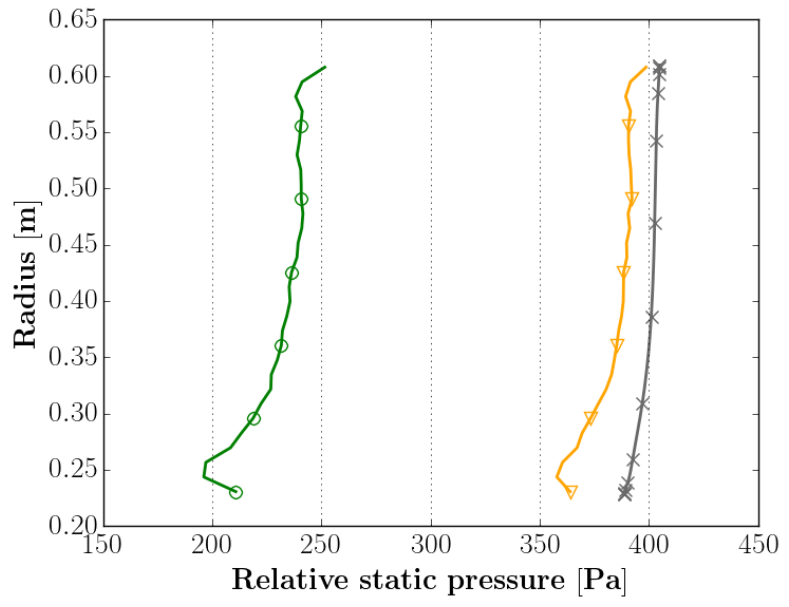

(b) Relative static pressure.

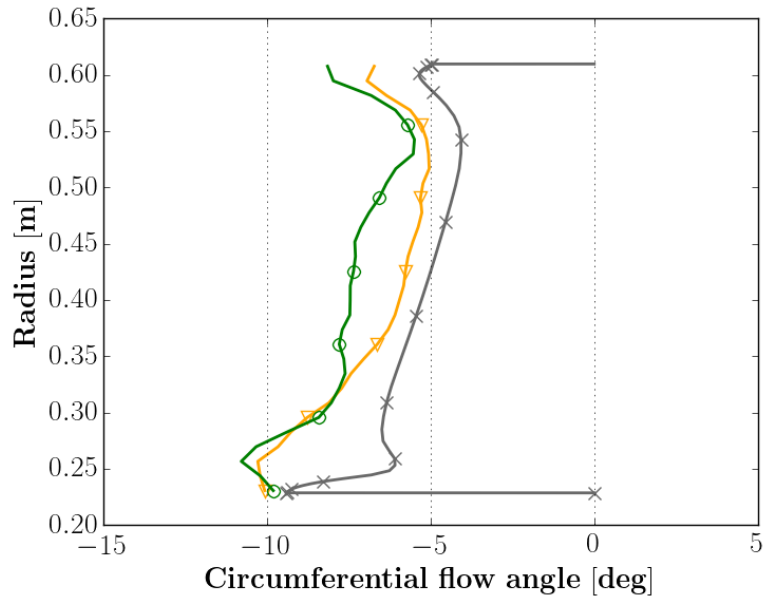

(d) Circumferential flow angle.

Fig. 10 Mean radial flow profiles extracted one chord downstream of the stator: comparison with RANS results. 
rearward arc with a radius of about $3.66 \mathrm{~m}$ from the exit plane as shown in Fig. 11. Pressure signals were acquired during $20 \mathrm{~s}$ with 256 samples per fan revolution. In the simulations, probes have been placed at the same locations to perform direct far-field noise comparisons. The signals are extracted 1632 times per rotation during the 10 last rotations (for a corresponding physical time of $0.33 \mathrm{~s}$ ).

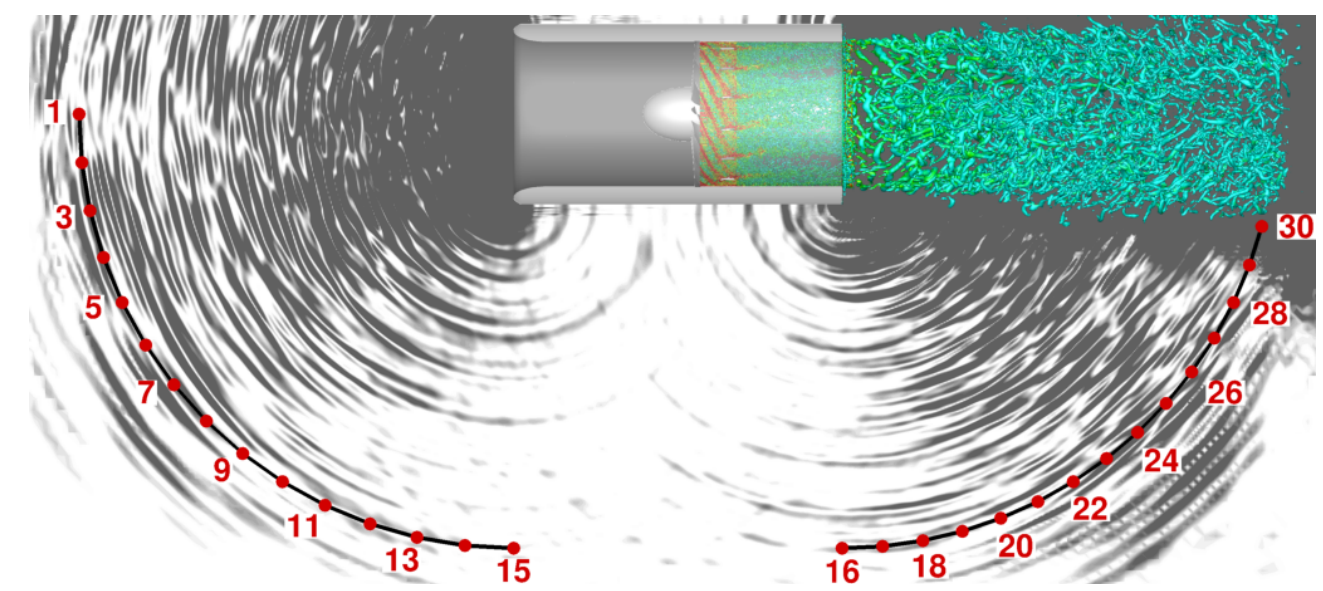

Fig. 11 Position of the far-field microphones in the horizontal plane $z=0 \mathrm{~m}$. One every two microphones is denoted with its number for clarity.

Power Spectral Density (PSD) of pressure fluctuations are computed on each probe using Welch's method with 8 blocks and $50 \%$ overlapping (leading to a frequency resolution $\Delta f$ of $13.5 \mathrm{~Hz}$ ) and are compared with the experimental PSD available in the database. Note that because of the average of the Welch's method, the tones are smoothed and their absolute levels are not reliable. The focus is therefore only put on the broadband spectrum in this part. The results obtained for the microphone \#8 with the three simulations are plotted in Fig. 12 for evaluation of the mesh resolution effect. The maximum frequency in the experimental data is $3840 \mathrm{~Hz}$ (corresponding to 8BPF) so that the spectrum are shown up to $4000 \mathrm{~Hz}$. Below $500 \mathrm{~Hz}$, the effect of mesh resolution is not clear and the levels predicted by the simulations are about $10 \mathrm{~dB}$ higher than the experimental ones. From $500 \mathrm{~Hz}$ to $3000 \mathrm{~Hz}$, the noise levels are globally overestimated by 5 to $10 \mathrm{~dB}$ with the coarse simulation. Refining the mesh tends to decrease these levels by 5 to $10 \mathrm{~dB}$ above $1000 \mathrm{~Hz}$ for the medium simulation and above $500 \mathrm{~Hz}$ for the fine simulation. As a result, the PSD spectrum predicted by the fine simulation is in good agreement with the experimental one in this frequency range. This comparison also demonstrates the capability of the LBM to accurately capture acoustics at high frequencies with a low computational cost. A similar behavior is observed for the other microphones so that only the results obtained with the fine simulation are now discussed for the sake of conciseness.

The PSD obtained at one every four microphones are given in Fig. 13 for the fine simulation and the measurements. For upstream microphones and low polar angles (up to microphone \#10), the overall agreement is excellent in the whole frequency range. At high polar angles however, the high frequencies (after $2000 \mathrm{~Hz}$ ) become underestimated, which is 


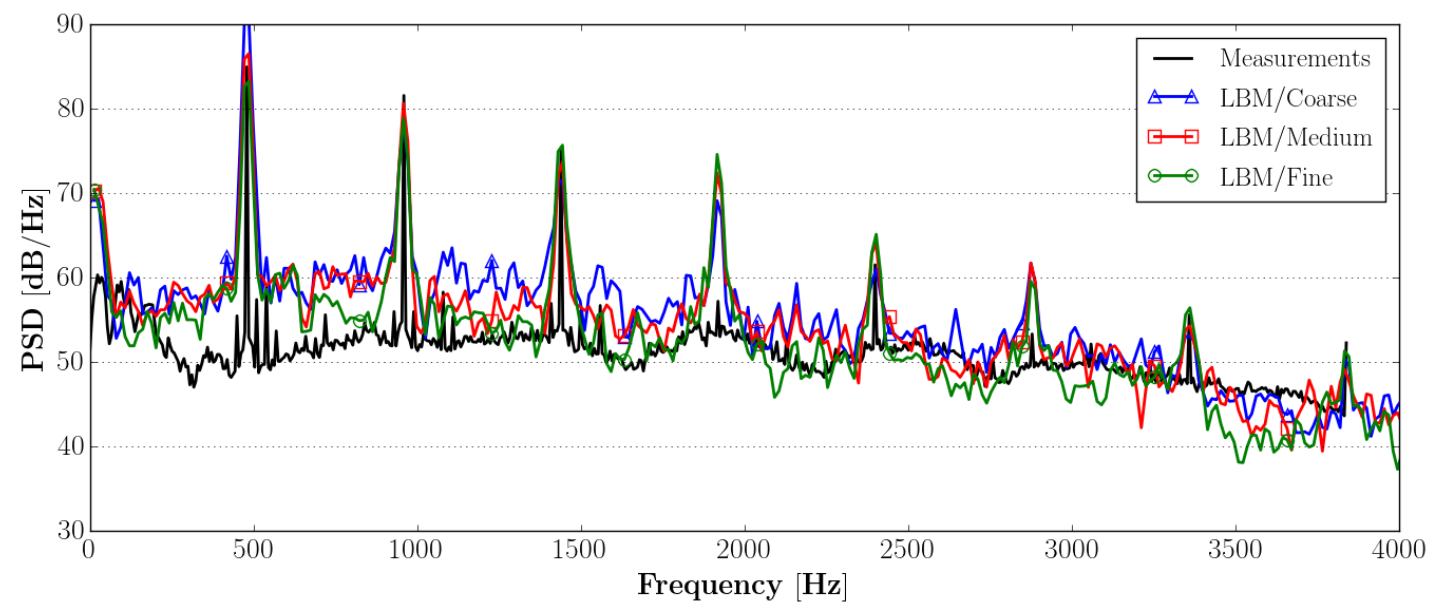

Fig. 12 PSD on the microphone \#8: effect of mesh refinement and comparison with experiments.

seen here on microphone \#14 with a a gap going up to $10 \mathrm{~dB}$. Nevertheless, this is expected to have only a little effect on the acoustic power given the low noise levels at these angles (around $40 \mathrm{~dB}$ ). For downstream microphones, the agreement is also very good at mid and high frequencies (after $1000 \mathrm{~Hz}$ ). The only exception is for microphone \#29 where the estimated noise levels become lower than the measured ones after $1500 \mathrm{~Hz}$. For all downstream angles, the low frequencies (below $1000 \mathrm{~Hz}$ ) tend to be overestimated by 10 to $15 \mathrm{~dB}$ in the simulations. The origin of this overprediction is not clearly established, but it is probably a combination of statistical errors (the statistics are made over 500 rotor rotations in the measurements when only 10 rotations are used in the simulation) and numerical errors due to reflections on the far-field boundaries for the lowest frequencies (the largest cell size is about half the wavelenght associated to the BPF). The proximity of the jet for the highest polar angles may also be responsible for hydrodynamic fluctuations that can pollute the measured sound pressure at these frequencies.

\section{Direct Far-Field Power Spectra}

The acoustic power is a relevant quantity for a practical characterization of sound sources. The pressure spectra shown up to now are indeed dependent on the source and observer positions and therefore do not directly provide a conservative quantity, as is the sound power evaluated below, to assess the noise at source.

The inlet and outlet radiated power will be considered separately here by integrating the normal component of the acoustic intensity over two distinct far-field surfaces. Since the flow is at rest in the far-field, the normal component of the acoustic intensity $I(\boldsymbol{x}, \omega)$ at position $\boldsymbol{x}$ of spherical coordinates $(r, \theta, \phi)$ and at pulsation $\omega$ is defined by:

$$
I(\boldsymbol{x}, \omega)=\frac{|p(\boldsymbol{x}, \omega)|^{2}}{\rho_{0} c_{0}}
$$

where $\rho_{0}$ and $c_{0}$ are the mean density and speed of sound of the stagnant fluid and $p(\boldsymbol{x}, \omega)$ is the acoustic pressure field 


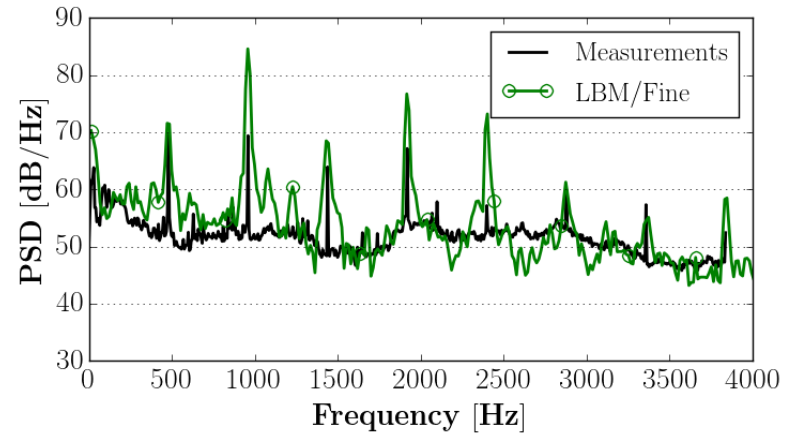

(a) Microphone \#2 (upstream).

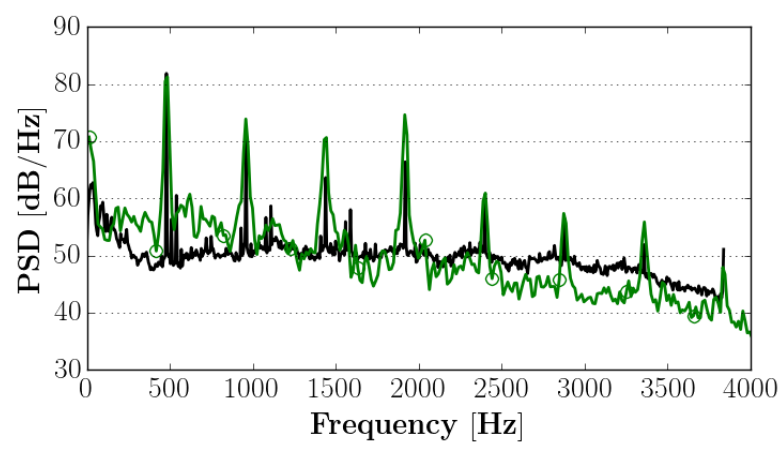

(c) Microphone \#10 (upstream).

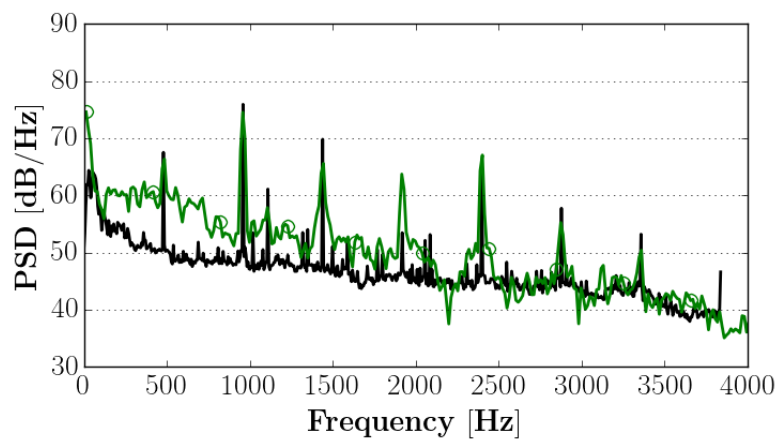

(e) Microphone \#17 (downstream).

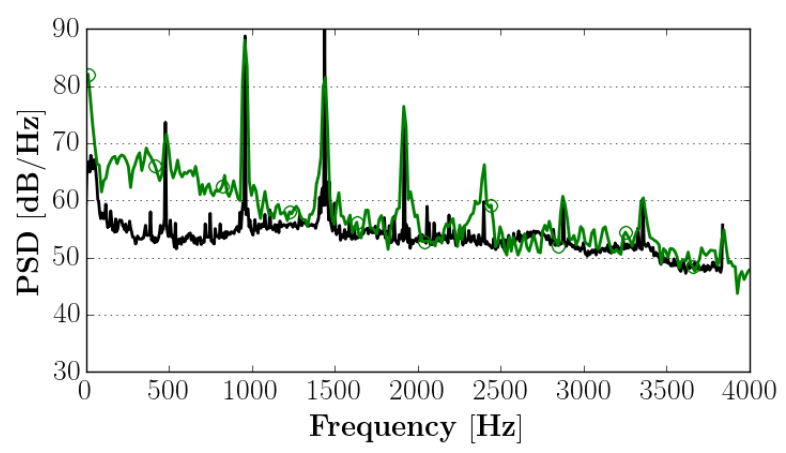

(g) Microphone \#25 (downstream).

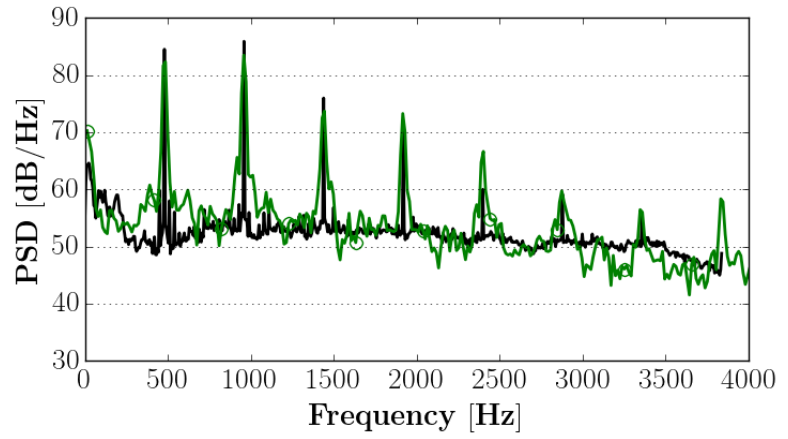

(b) Microphone \#6 (upstream).

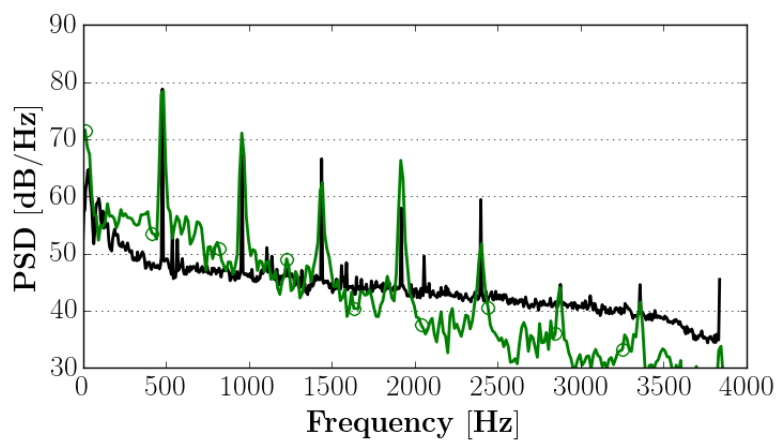

(d) Microphone \#14 (upstream).

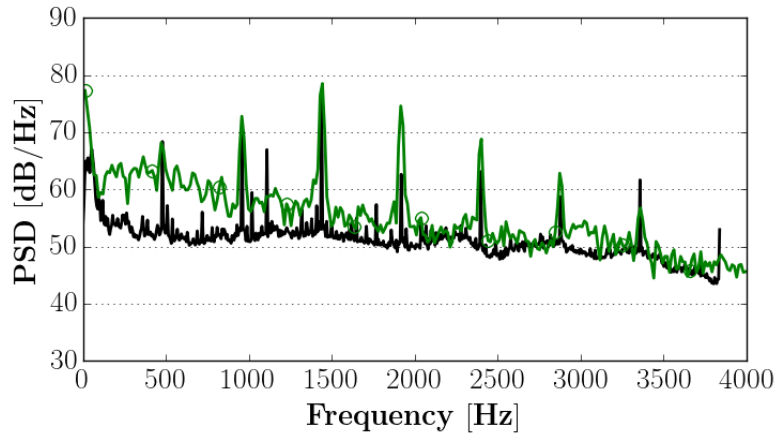

(f) Microphone \#21 (downstream).

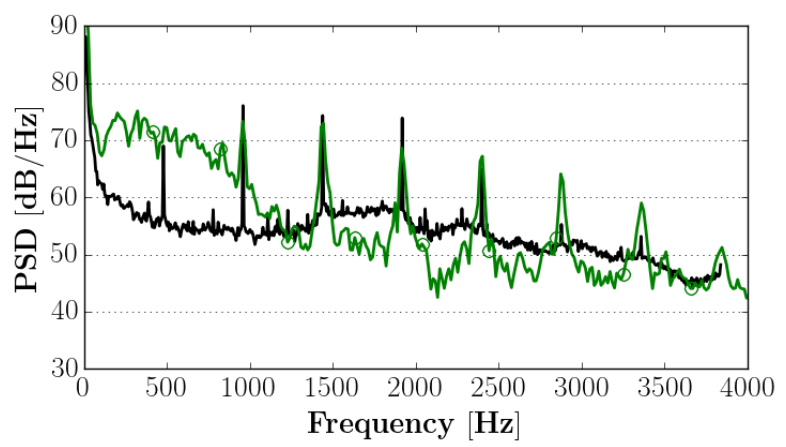

(h) Microphone \#29 (downstream).

Fig. 13 PSD on the far-field microphones: comparison of the fine simulation results with experiments. 
at the same pulsation. Given the axisymmetry of the configuration, the acoustic power $W(\omega)$, defined as the integral of the acoustic intensity, can be evaluated from the microphone arcs only:

$$
W(\omega)=2 \pi r^{2} \int_{\phi} I\left(r, \theta=0^{\circ}, \phi, \omega\right) \sin (\phi) d \phi,
$$

with $r$ the microphone arc radius and $\phi$ the polar angle defined from $0^{\circ}$ at the inlet to $180^{\circ}$ at the outlet. The surface defined by the revolution of the microphone arcs is not a closed surface so that the acoustic power could be underestimated in theory. However, the radiated noise levels are expected to be very low in the polar angles that are neglected (above $90^{\circ}$ for upstream noise; below $90^{\circ}$ and above $165^{\circ}$ for downstream noise) and are only due to some refraction effect by the mean flow and the nacelle lips. In addition, the acoustic power is evaluated from the experimental microphones and the numerical probes in the same way to have the most faithful comparisons. The resulting upstream and downstream power levels are depicted in Fig. 14. The effect of mesh resolution is clearly marked on these curves, with lower noise levels when refining. The acoustic power is reduced by $5 \mathrm{~dB}$ in average in the fine simulation when compared to the coarse simulation. The levels obtained with the fine mesh are very close to the experimental values in the mid- and high-frequency range, thus showing the capabilities of the LBM to correctly predict the broadband noise. Below 1000 $\mathrm{Hz}$ for the inlet and $1500 \mathrm{~Hz}$ for the outlet, the broadband noise levels are however overestimated in the simulation. The difference is around 5 to $10 \mathrm{~dB}$ in the inlet and around 10 to $15 \mathrm{~dB}$ in the outlet a these frequencies. This behavior is to be related to the low-frequency overestimation of the pressure spectra mentioned previously.

\section{Comparison with Indirect In-Duct Power Spectra}

The above results show that the broadband noise is a priori well captured by the LBM fine simulation at mid and high frequencies and at most positions. In order to strengthen these results, the capabilities of the present method to correctly propagate acoustic waves is evaluated in addition in this section to ensure that no spurious noise is generated across the refinement interfaces. This is done by comparing them with an indirect approach where the acoustic sources are extracted from the LBM simulations and are propagated analytically in the duct using Goldstein's analogy [32]. When considering the dipole sources as the dominant sources (reasonable assumption except at transonic regimes), the latter gives an expression of the acoustic field inside a semi-infinite straight duct of circular or annular (direct extension of the analogy) cross-section and crossed by a uniform and axial flow as a function of the rotor blade or stator vane unsteady loadings. In the following, $\rho_{0}, c_{0}$ and $M_{0}$ denote the density, speed of sound and axial Mach number of the in-duct uniform flow. The pressure field $p(\boldsymbol{x}, \omega)$ at position $\boldsymbol{x}$ of cylindrical coordinates $(x, r, \theta)$ and at pulsation $\omega$ therefore writes:

$$
p(\boldsymbol{x}, \omega)=\iint_{S} p(\boldsymbol{y}, \omega) n_{i}(\boldsymbol{y}) \frac{\partial G(\boldsymbol{x} \mid \boldsymbol{y}, \omega)}{\partial y_{i}} d S(\boldsymbol{y}),
$$




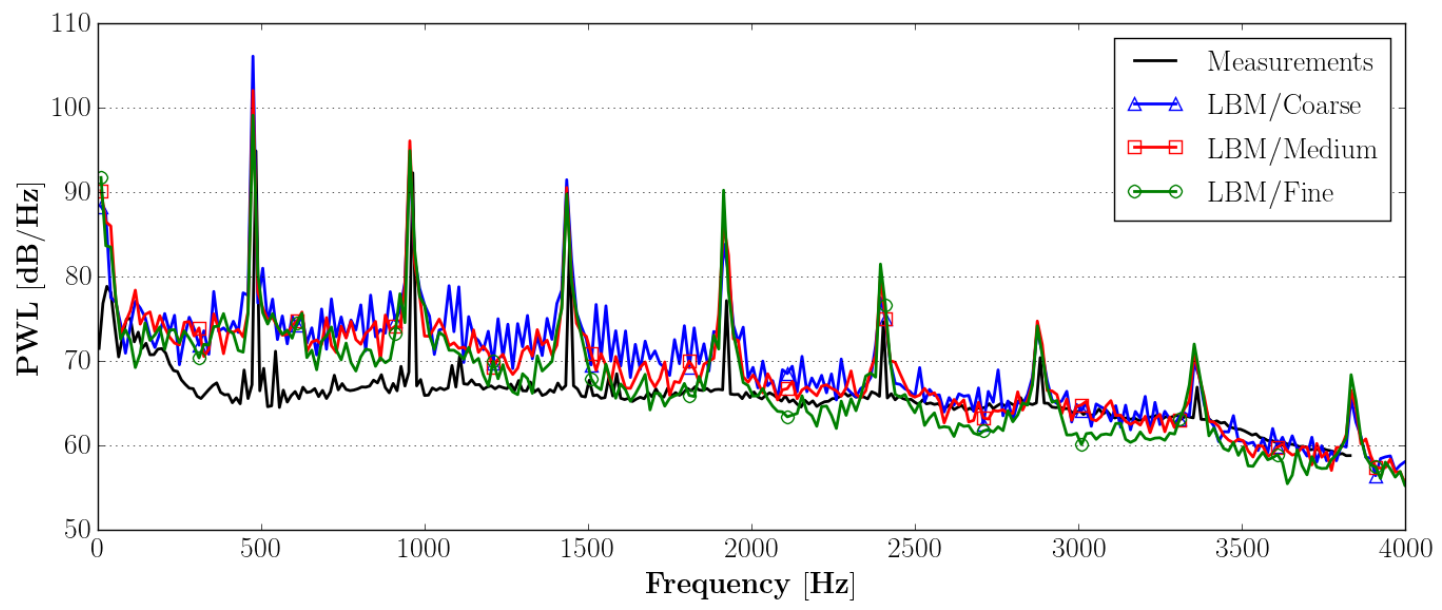

(a) Upstream.

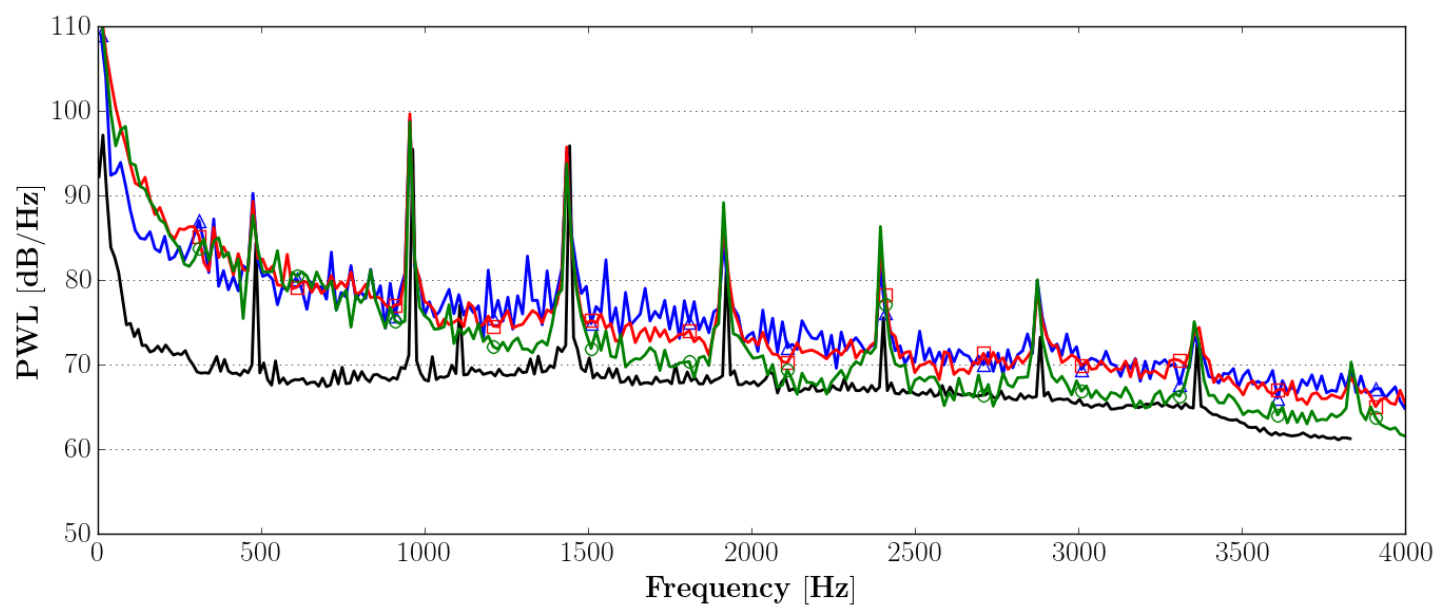

(b) Downstream.

Fig. 14 Acoustic power: effect of mesh refinement and comparison with experiments. 
where the integration is performed over every point $\boldsymbol{y}=\left(x_{y}, r_{y}, \theta_{y}\right)$ of normal $\boldsymbol{n}(\boldsymbol{y})=\left(n_{x}, n_{r}, n_{\theta}\right)$ of the source surface $S . G(\boldsymbol{x} \mid \boldsymbol{y}, \omega)$ is the in-duct Green's function expressed in the frequency domain as:

$$
G(\boldsymbol{x} \mid \boldsymbol{y}, \omega)=\frac{i}{2} \sum_{m=-\infty}^{+\infty} \sum_{n=1}^{+\infty} \frac{\overline{\psi_{m n}}(r, \theta) \psi_{m n}\left(r_{y}, \theta_{y}\right)}{\Gamma_{m n} k_{m n}} e^{-i \gamma_{m n}\left(x-x_{y}\right)}
$$

The symbol ${ }^{-}$denotes the complex conjugate. The functions $\psi_{m n}$ are known as the duct modes of azimuthal order $m$ and radial order $n$ and write:

$$
\psi_{m n}(r, \theta)=\left[A_{m n} J_{m}\left(\alpha_{m n} r\right)+B_{m n} Y_{m}\left(\alpha_{m n} r\right)\right] e^{-i m \theta}
$$

with $J_{m}$ and $Y_{m}$ the Bessel functions of order $m$ of the first and second kinds respectively. The coefficients $A_{m n}$ and $B_{m n}$ and the radial wave number $\alpha_{m n}$ are determined from the boundary conditions, written for hard walls:

$$
\left.\frac{\partial \psi_{m n}}{\partial r}\right|_{R_{h}, R_{t}}=0
$$

where $R_{h}$ and $R_{t}$ denote the hub and tip duct radius respectively. $\Gamma_{m n}$ is the norm of the modal functions $\psi_{m n}$ :

$$
\Gamma_{m n}=\int_{0}^{2 \pi} \int_{R_{h}}^{R_{t}}\left|\psi_{m n}(r, \theta)\right|^{2} r d r d \theta
$$

$k_{m n}$ is defined by $k_{m n}^{2}=k^{2}-\beta_{0}^{2} \alpha_{m n}^{2}$ with $k=\omega / c_{0}$ the acoustic wavenumber and $\beta_{0}=\sqrt{1-M_{0}^{2}}$ the reciprocal Lorentz factor of the flow and $\gamma_{m n}$ is the axial wave number:

$$
\gamma_{m n}=\frac{M_{0} k}{\beta_{0}^{2}} \pm \frac{k_{m n}}{\beta_{0}^{2}}
$$

The upper sign (here +) and the lower sign (here -) stand for the upstream and downstream propagation respectively. Note that, for clarity, the dependency of $k, k_{m n}$ and $\gamma_{m n}$ on the pulsation $\omega$ is not written explicitely.

In this framework, the acoustic intensity is computed using Cantrell and Hart's formulation [33] to account for the presence of the flow (unlike in Eq. (20)) and the acoustic power is obtained by integrating the axial component of the intensity over the duct cross sections. The resulting power $W(\omega)$ at pulsation $\omega$ can be expressed as the sum of the acoustic power carried by each mode [32, 34]:

$$
W(\omega)=\mp 2 \sum_{m=-\infty}^{+\infty} \sum_{n=1}^{+\infty} \Gamma_{m n} \operatorname{Re}\left[\left(1+M_{0}^{2}\right) p_{m n}(\omega) \overline{u_{m n}(\omega)}+\frac{M_{0}}{\rho_{0} c_{0}}\left|p_{m n}(\omega)\right|^{2}+\rho_{0} c_{0} M_{0}\left|u_{m n}(\omega)\right|^{2}\right] .
$$

Re represents the real part operator and $p_{m n}(\omega)$ and $u_{m n}(\omega)$ are defined as the modal pressure and modal axial velocity 
respectively, such that the pressure field $p(\boldsymbol{x}, \omega)$ and the axial velocity field $u(\boldsymbol{x}, \omega)$ at pulsation $\omega$ write:

$$
p(\boldsymbol{x}, \omega)=\sum_{m=-\infty}^{+\infty} \sum_{n=1}^{+\infty} p_{m n}(\omega) \overline{\psi_{m n}}(r, \theta) e^{-i \gamma_{m n} x}, \quad u(\boldsymbol{x}, \omega)=\sum_{m=-\infty}^{+\infty} \sum_{n=1}^{+\infty} u_{m n}(\omega) \overline{\psi_{m n}}(r, \theta) e^{-i \gamma_{m n} x} .
$$

Using Eqs. 221 and (23) gives:

$$
p_{m n}(\omega)=\frac{1}{2} \frac{S_{m n}(\omega)}{\Gamma_{m n} k_{m n}}
$$

with:

$$
S_{m n}(\omega)=i \iint_{S} p(\boldsymbol{y}, \omega) n_{i}(\boldsymbol{y}) \frac{\partial\left\{\psi_{m n}\left(r_{y}, \theta_{y}\right) e^{i \gamma_{m n} x_{y}}\right\}}{\partial y_{i}} d S(\boldsymbol{y})
$$

Finally, the projection over the axial direction of the momentum conservation equation yields:

$$
u_{m n}(\omega)=\left(\frac{-M_{0} k \mp k_{m n}}{k \pm M_{0} k_{m n}}\right) \frac{p_{m n}(\omega)}{\rho_{0} c_{0}} .
$$

Combining the above equations gives the usual and much more practical expression for the acoustic power:

$$
W(\omega)=\frac{k \beta_{0}^{4}}{2 \rho_{0} c_{0}} \sum_{m=-\infty}^{+\infty} \sum_{\substack{n=1 \\ k_{m n}^{2} \geq 0}}^{+\infty} \frac{\left|S_{m n}(\omega)\right|^{2}}{\Gamma_{m n} k_{m n}\left(k \pm M_{0} k_{m n}\right)^{2}} .
$$

Note that the summation is limited to the cut-on modes that satisfy the condition $k_{m n}^{2} \geq 0$. Indeed, when this condition is not respected, $k_{m n}$ is purely imaginary and the mode $(m, n)$ becomes evanescent (or cut-off) and does not contribute to the acoustic power.

The in-house ONERA code FanNoise that solves the above equations is used to evaluate the upstream and downstream acoustic power by integrating the unsteady loadings on the stator vane surfaces extracted from the fine simulation. The contribution of the rotor on the noise is therefore not accounted for, but it is expected to be negligible as 1) there is no inflow distortion and 2) the self noise is minor at such rotation speeds. To provide enough statistics, the static pressure over the stator vane surface is extracted 1632 times per rotation during the 10 last rotations. The results are shown in Fig. 15 and are compared with both the measurements and the direct acoustic power computed as in Fig.14. For low frequencies (below $1000 \mathrm{~Hz}$ ), there are important differences (higher than $10 \mathrm{~dB}$ below $500 \mathrm{~Hz}$ ) between the direct and indirect predicted power levels. The analytical result necessarily tends to zero at low frequencies because all modes become theoretically cut-off while residual noise can appear in the direct predictions (other noise sources, residual modes) and the experiments (installation effects). In addition, the duct-end reflections that are neglected in the indirect approach (semi-infinite duct) can be important at these frequencies. Nevertheless, in the largest part of the frequency range, the comparison between both power prediction methods gives meaningful results. For outlet noise, the agreement between the direct power evaluation and the indirect one based on Goldstein's analogy is very good above $1000 \mathrm{~Hz}$. 
This result shows that the acoustic propagation is correctly captured by the LBM simulation. For the inlet noise however, there is a gap between both approaches, of about $3 \mathrm{~dB}$ between $1000 \mathrm{~Hz}$ and $2500 \mathrm{~Hz}$ and then becoming higher with the frequency. This can be explained by the rotor screen effect (sound scattering by the rotor) and the presence of swirling flow in the interstage, both neglected in the Goldstein's formulation and expected to be important for upstream propagation of acoustic sources located on stator vanes.

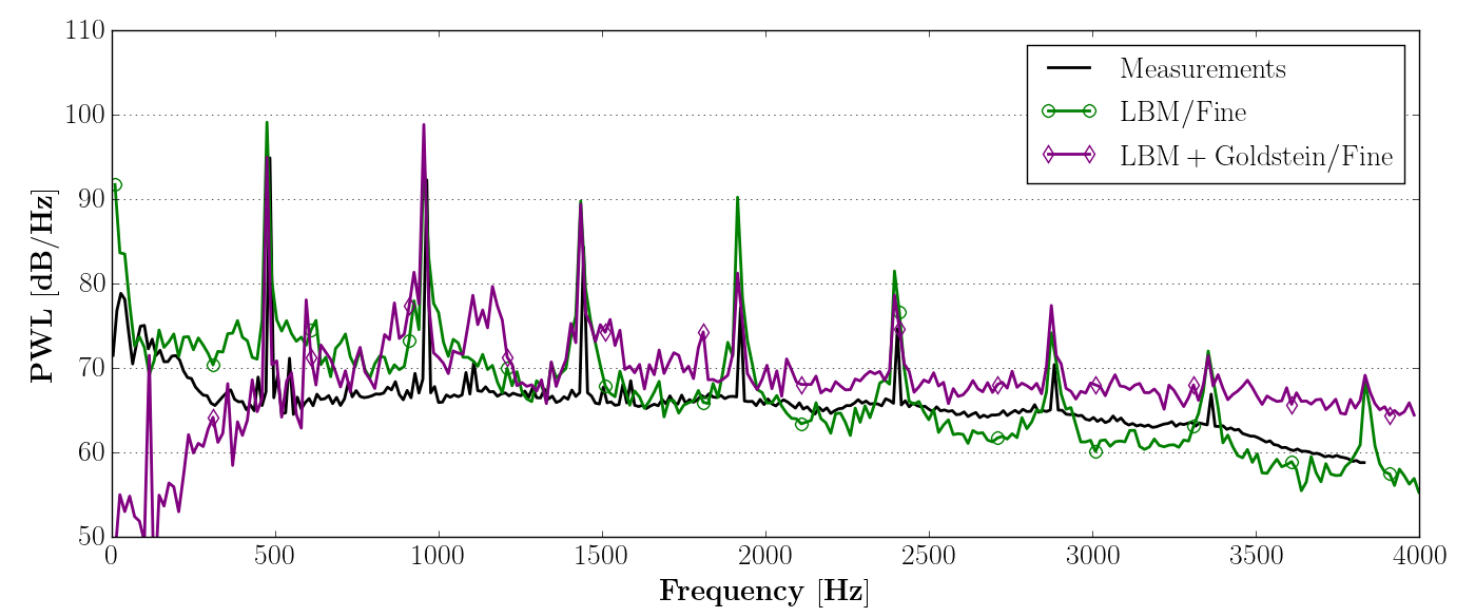

(a) Upstream.

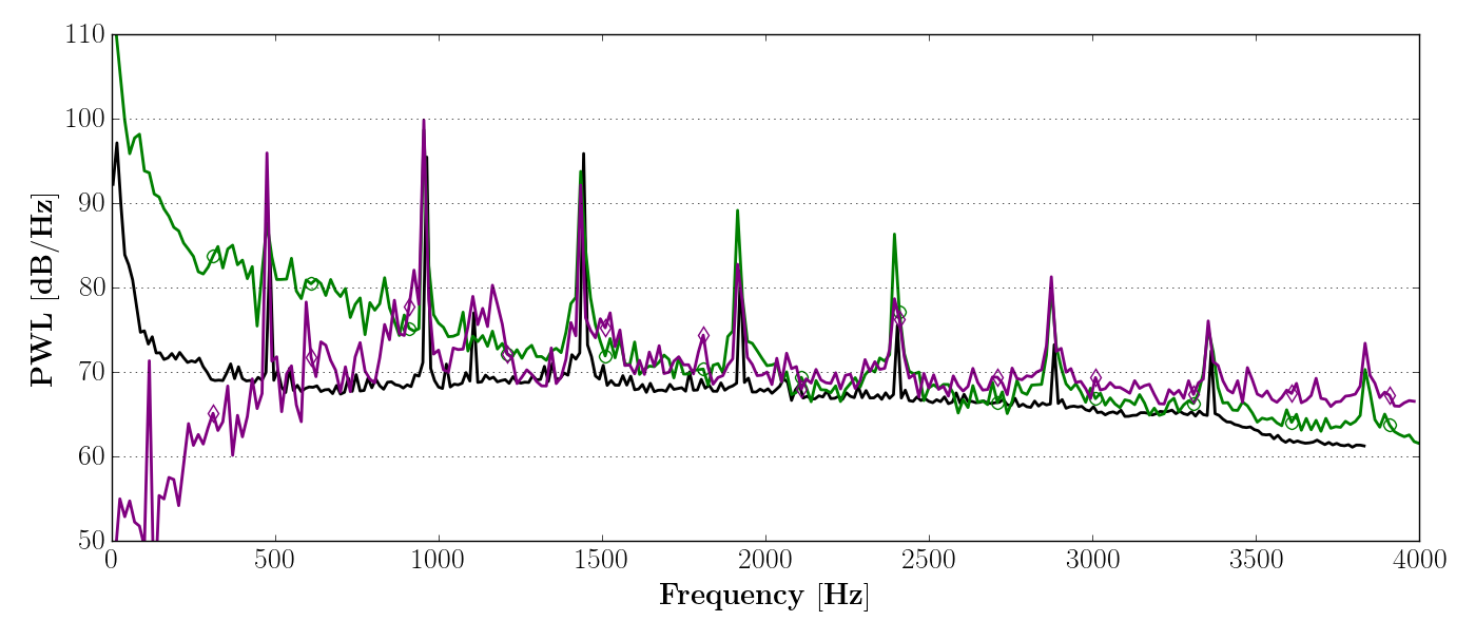

(b) Downstream.

Fig. 15 Acoustic power: comparison of direct and indirect formulation for the fine simulation.

\section{Direct In-Duct Acoustic Modes}

The final evaluation of the broadband noise results is conducted here by computing the acoustic modes directly from the pressure fluctuations extracted from the simulation. This is done by projection of the numerical pressure field 
$p(x, r, \theta, \omega)$ at pulsation $\omega$ over the duct modes:

$$
p_{m n}(\omega)=\frac{1}{\Gamma_{m n}} \int_{0}^{2 \pi} \int_{R_{h}}^{R_{t}} p(x, r, \theta, \omega) \psi_{m n}(r, \theta) r d r d \theta
$$

This procedure is applied for the first 50 rotation harmonics at two axial positions, one at the inlet and one at the outlet, corresponding to the locations of the rotating rakes in the experiments. The time Fourier transform of the pressure field $p(x, r, \theta, \omega)$ is computed from the static pressure extracted over these axial planes during one rotor rotation to limit the storage, so that the lowest frequencies may not be statistically converged. The results obtained with the fine simulation are presented in Fig. 16 in the form of a diagram showing the angular mode SPL per frequency and azimuthal order (by summing the contribution of each radial mode). It would have been possible to obtain a similar diagram by only performing a circumferential Fourier transform but the results would have been dependent on the radial position. By projecting on the radial modes, the contribution of all radii is accounted for. Note also that the angular mode SPL is different from the modal acoustic power, the latter being zero by definition for cut-off modes. The angular mode SPL is especially studied here to evaluate the capability of the present LBM simulations to capture the cut-on/cut-off transition. There is no corresponding experimental data (available data only in the form of modal power for the BPF tones) but the theoretical cut-on/cut-off transition computed using the equations of Sec. V.A.3 is added in dashed lines for comparison. The transition between cut-on and cut-off modes is clearly seen in the simulation and perfectly matches the theoretical one. The residual level of the cut-off modes is higher in the outlet plane, which can be explained by the presence of vorticity (i.e. non-acoustic) fluctuations in this region due to the presence of rotor blade and stator vane wakes. It can also be observed that the low frequencies (below rotation harmonic 16) are polluted with high levels in the cut-off region and this might be related to their low statistical convergence due to the limited time signal. Finally, the broadband noise is distributed over all cut-on modes with no particular pattern as expected. This is not the case of the tonal noise, corresponding to the rotation harmonics 16,32 and 48 for the $1 \mathrm{BPF}, 2 \mathrm{BPF}$ and $3 \mathrm{BPF}$ respectively (16 rotor blades), which will be the focus of next section.

\section{B. Tonal Noise}

\section{Direct Tonal Noise Directivity}

This section deals with tonal noise results. The tones are evaluated by computing Sound Pressure Levels (SPL) for the first three BPFs along the farfield microphone arcs using the data extracted over the last rotation. No averaging method is performed here in order to keep the exact BPF levels. The resulting tone directivities are presented in Fig. 17 for the three simulations and are compared with the experimental data. The polar angles going from $0^{\circ}$ to $90^{\circ}$ correspond to the forward arc $\left(0^{\circ}\right.$ being on the fan axis) while the angles between $90^{\circ}$ and $180^{\circ}$ correspond to the rearward arc. The effect of mesh refinement on the first three BPFs is not very clear: it decreases the noise levels at 


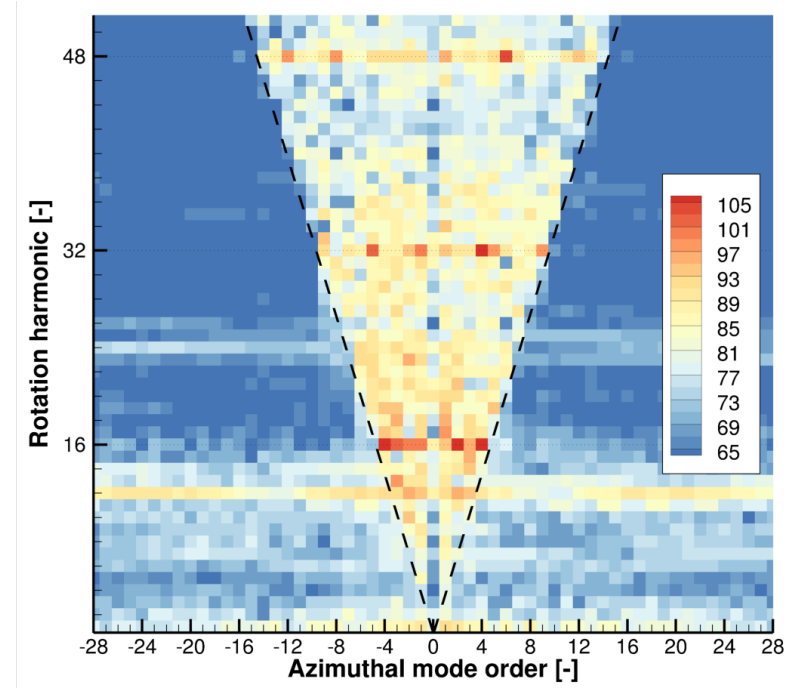

(a) Upstream.

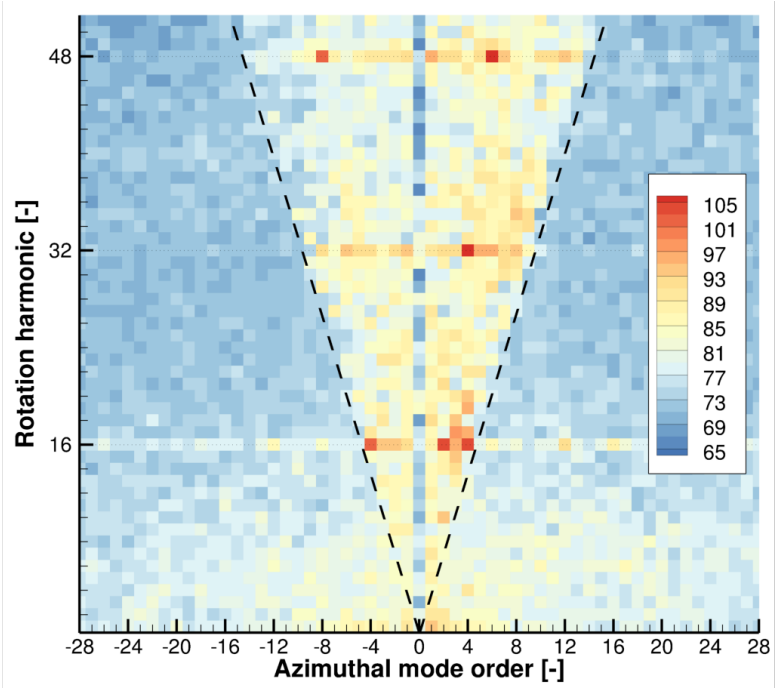

(b) Downstream.

Fig. 16 Angular mode SPL per frequency and azimuthal order for the fine simulation. The theoretical cuton/cut-off transition is added in dashed line.

some positions (upstream arc at 1BPF and downstream arc at 1BPF and 2BPF) while it increases them at some other positions (upstream arc at 2BPF). In all cases, important quantitative differences with the measurements are found. For the fine mesh, the noise levels are globally overestimated by 5 to $10 \mathrm{~dB}$ depending on the location and the frequency. These results illustrate how challenging is to capture the tonal noise (deterministic phenomenom) with an intrinsically unsteady method like the LBM when the acquisition time signal is limited (these difficulties are circumvented for broadband noise evaluation by the use of averaging techniques, such as the Welch's method). However, despite these quantitative differences, the directivity effects are reasonably well captured with the fine simulation, except for outlet noise at $1 \mathrm{BPF}$ where no particular shape emerges.

\section{Direct In-Duct Acoustic Modes}

The evaluation of tonal noise is completed with a duct mode analysis. Based on Tyler and Sofrin's (TS) formula, the interaction of rotor blade wakes with stator vanes will only cause the emergence of the modes with azimuthal order $m=s B-k V$, with $B$ the number of rotor blades, $V$ the number of stator vanes, $s$ the order of the considered BPF harmonic and where $k$ can be any integer [35]. In the present studied configuration, $B=16$ and $V=14$. The expected modes that lie in the cut-on range are therefore given by $m=2$ at 1BPF, $m=4$ at $2 \mathrm{BPF}$ and $m=6$ and $m=-8$ at 3BPF.

The capability of the present LBM simulations to correctly capture the expected modes is evaluated in this section. The same decomposition as the one described by Eq. (34) is performed, but the focus is only put on the BPF and its harmonics. The variable of interest here becomes the acoustic power carried by each mode (or modal power) to allow the comparison with the one derived from the rotating rake measurements [20]. It is computed using Cantrell and Hart's 


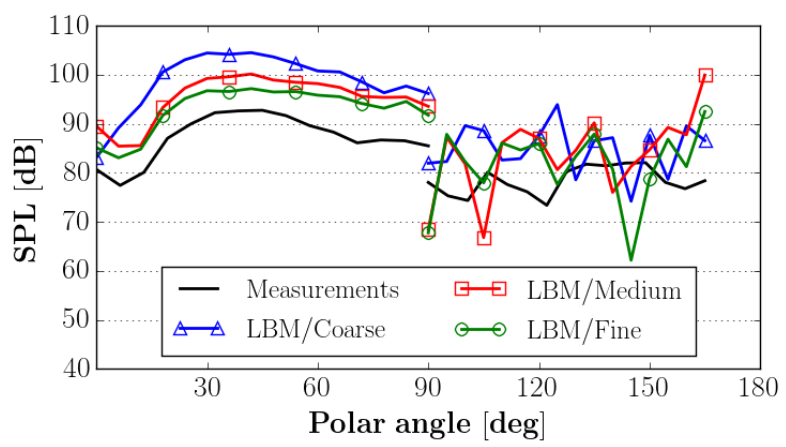

(a) BPF1.

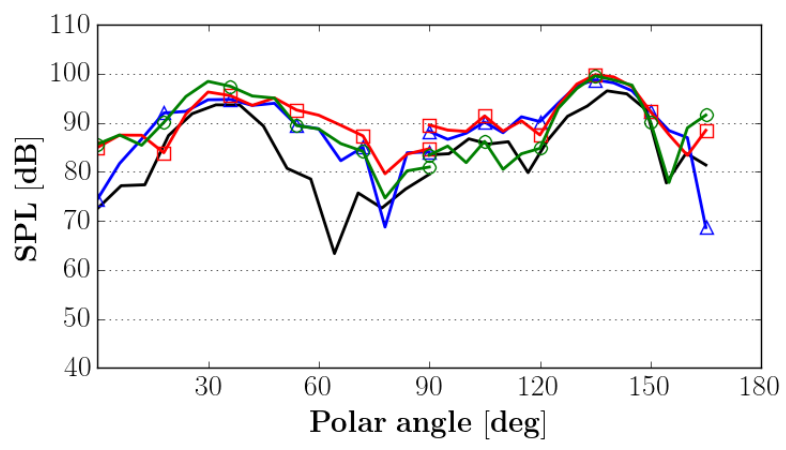

(b) BPF2.

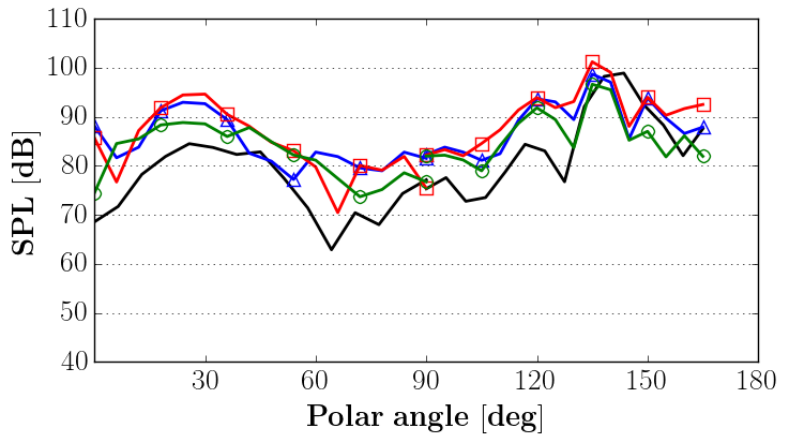

(c) BPF3.

Fig. 17 SPL directivity at the BPF and its harmonics: effect of mesh refinement and comparison with experiments. 
formulation of Eq. 28, with the modal pressure $p_{m n}(\omega)$ determined by Eq. 34 and the modal axial velocity $u_{m n}(\omega)$ determined from the modal pressure by Eq. (32). It is assumed that only upstream-propagative waves are present in the inlet and only downstream-propagative waves are present in the outlet by neglecting the reflections at both duct ends. The resulting levels are not identical to the ones of the modal SPL shown in Fig. 16 because the propagative efficiency of the modes is now accounted for. The results obtained with the fine simulation in the inlet plane for the first three BPFs are given in Fig. 18 and are compared with experimental rotating rake results. Only the cut-on modes are shown for each BPF as the power associated with cut-off modes is zero. In the experiments, the TS interaction modes are well captured (except the mode $m=-8$ at 3BPF) and clearly emerge with a power level above the other modes by 15 to $20 \mathrm{~dB}$. In the simulation, the TS modes are also the most energetic ones but their emergence is less clear with levels between $6 \mathrm{~dB}$ and $8 \mathrm{~dB}$ higher than the second most energetic mode. The outlet results are also given in Fig. 19 . In the experiments, the predominance of the interaction mode $m=2$ at $1 \mathrm{BPF}$ is not very pronounced because of the important energy carried by the mode $m=0$. At $2 \mathrm{BPF}$ and $3 \mathrm{BPF}$ however, the TS interaction modes are clearly predominant. The simulation mode distribution highlights a more marked emergence of the TS modes than in the inlet plane, especially at 2BPF and 3BPF. At 2BPF, the emergence of the TS mode $m=4$ is particularly marked with a level $15 \mathrm{~dB}$ higher than any other mode. At 3BPF, the predominance of the two TS modes is well captured with an emergence of $10 \mathrm{~dB}$ and 5 $\mathrm{dB}$ with respect to the third most energetic mode. In conclusion, even if the tone noise levels are overestimated by the fine simulation, the emergence of the expected interaction modes is well recovered. It is less pronounced 1) at the inlet plane, probably because the acoustic waves coming from the stator vanes are crossing twice a mobile/fix interface which might introduce some spurious modes and 2) at 1BPF for both inlet and outlet planes, which might be related to the previously mentioned difficulties to get reliable low-frequency noise levels.

\section{Conclusion}

The capability of isothermal LBM to correctly predict the aerodynamics and acoustics of a low-speed turbofan has been evaluated in this paper. The assessment has been done on the ANCF model whose fan-blade tip Mach number of 0.34 at nominal speed is a priori suited for the method. The LBM simulations have been performed using the ProLB solver and three mesh resolutions (coarse, medium, fine) have been evaluated. The comparison with the experiments has only been done at the nominal regime of $1800 \mathrm{rpm}$ and with a stator of 14 vanes spaced from the rotor by one chord, as it is the most instrumented configuration. An additional case corresponding to a higher regime (2000 rpm) and with 14 stator vanes spaced from the rotor by 0.27 rotor chord has also been simulated with the fine mesh resolution to compare with RANS results available in the NASA ANCF database.

The aerodynamic results have first been extensively studied, and a similar underprediction of the fan performance values as the one reported by the previous related studies has been recovered. A detailed analysis of the aerodynamic profiles inside the turbofan has been led and has revealed a likely uncertainty of the absolute values of some variables 


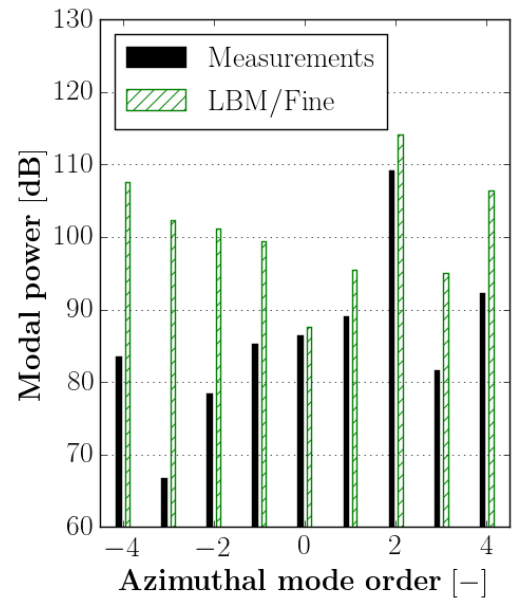

(a) $1 \mathrm{BPF}$.

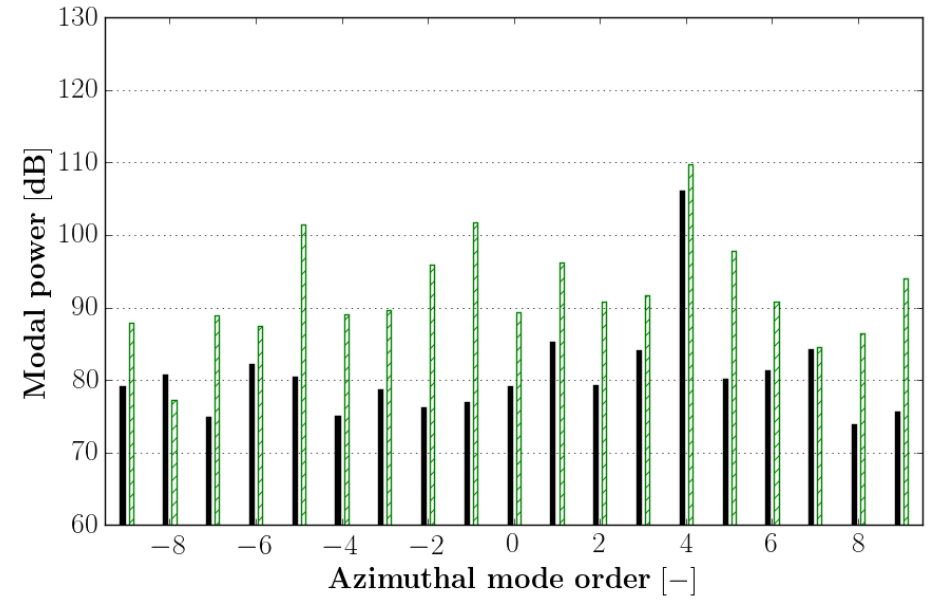

(b) $2 \mathrm{BPF}$.

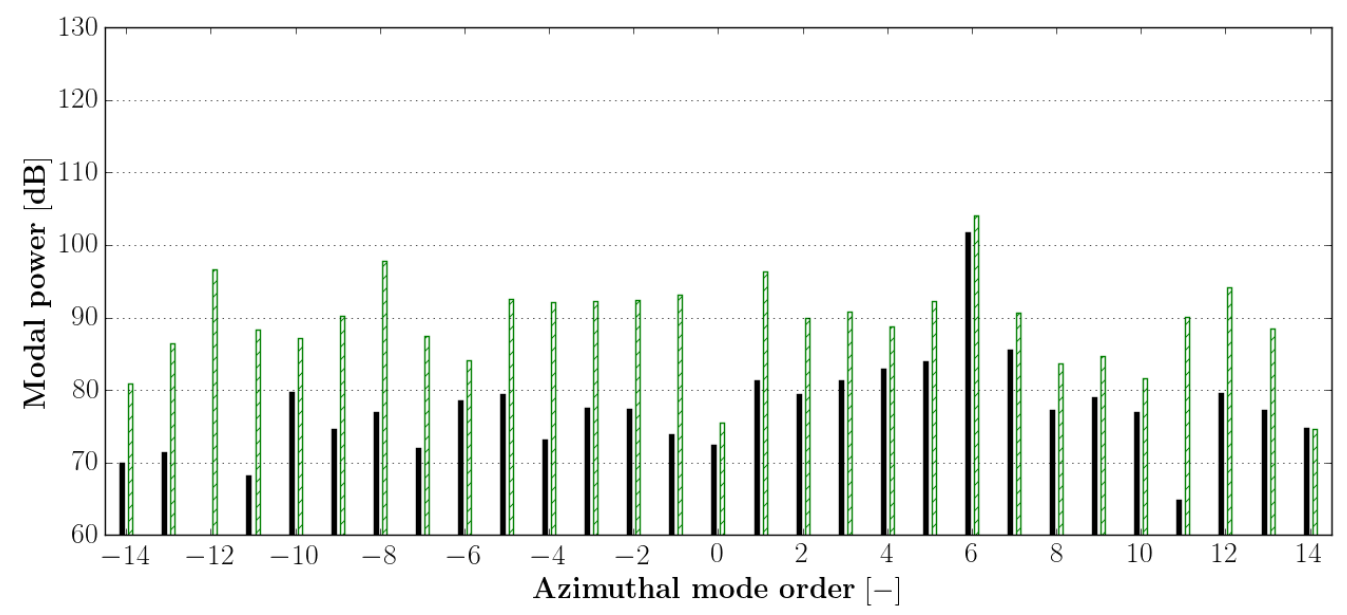

(c) 3BPF.

Fig. 18 Upstream modal power: comparison of the fine simulation results with experiments. 


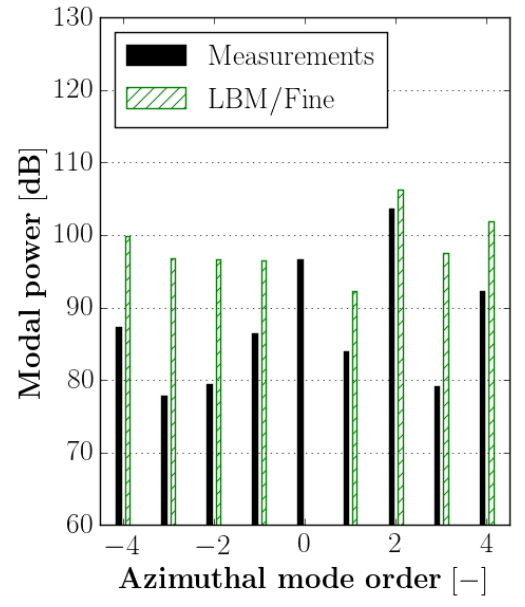

(a) $1 \mathrm{BPF}$.

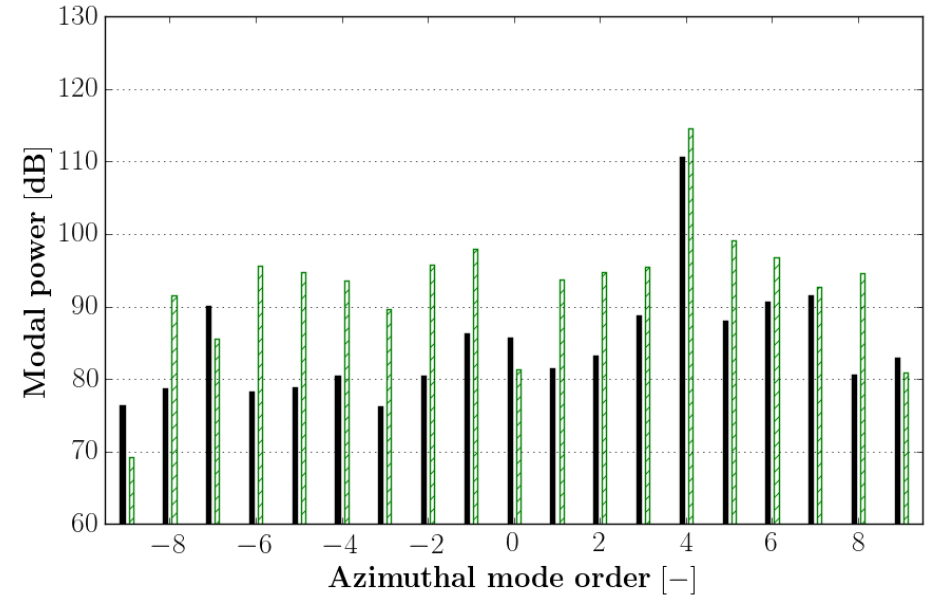

(b) $2 \mathrm{BPF}$.

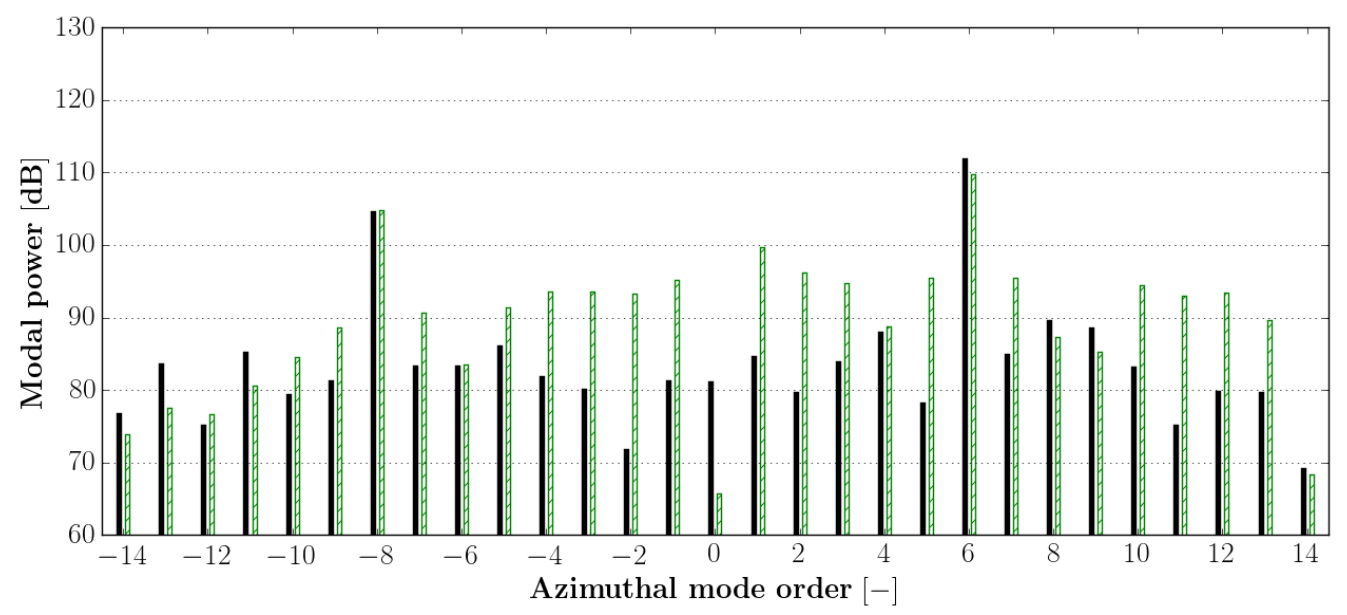

(c) 3BPF.

Fig. 19 Downstream modal power: comparison of the fine simulation results with experiments. 
in the experimental data. Only relative comparisons have therefore been performed with the simulations and show a good agreement. To further evaluate the aerodynamics, especially in terms of absolute values, the database RANS solution has been compared with the LBM simulation of the same configuration. Both methods compared very well in terms of fan performance, inlet radial flow profiles and outlet radial flow profiles. The RANS methodology being well suited for mean flow prediction, these comparisons validate the aerodynamic results of the performed isothermal LBM simulations.

The acoustic results have then been thoroughly investigated. The focus has been put on direct noise evaluation, by taking advantage of the capacity of the LBM solver to accurately propagate acoustic waves with a limited spatial resolution. The far-field broadband noise directly extracted from the simulation has therefore been compared with the measurements and an excellent agreement has been obtained for the fine simulation, despite overpredicted levels at low frequencies. The capability of the isothermal LBM to correctly propagate acoustic waves has been further demonstrated by successfully comparing the direct noise prediction with indirect ones based on Goldstein's analogy. Finally, the tonal noise results have been assessed. Even with the fine mesh, the tone levels predicted by the simulation were overestimated by 5 to $10 \mathrm{~dB}$ in average. This is probably to be related to the difficulty to get a reliable tonal signature with a fully unsteady method like the LBM when the acquisition time is limited and the statistical errors are important. Nevertheless, despite these differences in terms of absolute levels, the directivity patterns and the expected interaction modes are reasonably recovered.

All these results have demonstrated the capability of the isothermal LBM to properly evaluate the aerodynamics and the acoustics (essentially the broadband contribution) of a low-speed turbofan. To the author's knowledge, this is the first time that such a validation of the isothermal LBM on both turbofan aerodynamics and acoustics is published. Even if thermal LBM for compressible flows exist and are currently extensively studied by the LBM community, the isothermal LBM formulation and its impressive efficiency still presents a huge interest for low-Mach aeroacoustic applications. The direct philosophy adopted in the paper, which consists in computing in a single simulation the turbulent wakes emitted from the rotor blades, their advection in the interstage, their impact on the stator vanes and the propagation of the resulting acoustic waves up to the far-field, has been clearly demonstrated by the use of the LBM formalism. The low-dissipative characteristics of the method and the intrinsic local time stepping indeed allow the acoustic waves to be propagated over long distances. One last advantage of the method, which is inherent to the use of a lattice that makes mandatory the implementation of immersed boundary conditions, is that it is perfectly suited for parameterisation study. One significant illustration of this aspect: only a few minutes were needed to change the position of the stator and the rotation speed for comparison against the RANS results. This aspect could be particularly appropriated for the study of noise reduction technologies, such as serrations or swept vanes, and for evaluating complex geometries with inhomegeneous and unevenly spaced stator vanes. 


\section{Acknowledgments}

The authors would like to thank D. Sutliff from NASA Glenn Research Center for 1) providing the geometry and the experimental results of the Advanced Noise Control Fan and 2) his help in the interpretation of the experimental data. The simulations presented in the paper have been performed using the ProLB solver (http://www.prolb-cfd.com/) and most of the post-processing has been achieved using the Cassiopée Python library (http://elsa.onera.fr/Cassiopee/).

\section{References}

[1] Khorrami, M. R., Fares, E., and Casalino, D., “Towards Full Aircraft Airframe Noise Prediction: Lattice Boltzmann Simulations,” 20th AIAA/CEAS Aeroacoustics Conference, 2014. https://doi.org/10.2514/6.2014-2481.

[2] Sengissen, A., Giret, J.-C., Coreixas, C., and Boussuge, J.-F., "Simulations of LAGOON landing-gear noise using LatticeBoltzmann Solver,” 21st AIAA/CEAS Aeroacoustics Conference, 2015. https://doi.org/10.2514/6.2015-2993

[3] Lévêque, E., Touil, H., Malik, S., Ricot, D., and Sengissen, A., "Wall-modeled large-eddy simulation of the flow past a rod-airfoil tandem by the Lattice Boltzmann method," International Journal of Numerical Methods for Heat \& Fluid Flow, Vol. 28, No. 5, 2018, pp. 1096-1116. https://doi.org/10.1108/HFF-06-2017-0258

[4] Casalino, D., Avallone, F., Gonzalez-Martino, I., and Ragni, D., "Aeroacoustic study of a wavy stator leading edge in a realistic fan / OGV stage," Journal of Sound and Vibration, Vol. 442, 2019, pp. 138-154. https://doi.org/10.1016/j.jsv.2018.10.057

[5] Noh, H.-M., "Numerical analysis of aerodynamic noise from pantograph in high-speed trains using lattice Boltzmann method," Advances in Mechanical Engineering, Vol. 11, No. 7, 2019, p. 1687814019863995. https://doi.org/10.1177/1687814019863995

[6] Hou, Y., Angland, D., Sengissen, A., and Scotto, A., "Lattice-Boltzmann and Navier-Stokes Simulations of the Partially Dressed, Cavity-Closed Nose Landing Gear Benchmark Case," 25th AIAA/CEAS Aeroacoustics Conference, 2019. https: //doi.org/10.2514/6.2019-2555

[7] Romani, G., and Casalino, D., "Rotorcraft blade-vortex interaction noise prediction using the Lattice-Boltzmann method," Aerospace Science and Technology, Vol. 88, 2019, pp. 147-157. https://doi.org/10.1016/j.ast.2019.03.029

[8] Avallone, F., Ragni, D., and Casalino, D., "On the effect of the tip-clearance ratio on the aeroacoustics of a diffuser-augmented wind turbine," Renewable Energy, Vol. 152, 2020, pp. 1317-1327. https://doi.org/10.1016/j.renene.2020.01.064

[9] Romani, G., Ye, Q., Avallone, F., Ragni, D., and Casalino, D., "Numerical analysis of fan noise for the NOVA boundary-layer ingestion configuration," Aerospace Science and Technology, Vol. 96, 2020, p. 105532. https://doi.org/10.1016/j.ast.2019.105532

[10] Chen, S., and Doolen, G. D., "Lattice Boltzmann Method for Fluid Flows," Annual Review of Fluid Mechanics, Vol. 30, 1998, pp. 329-364. https://doi.org/10.1146/annurev.fluid.30.1.329. 
[11] Horstmann, J. T., Le Garrec, T., Mincu, D.-C., and Lévêque, E., "Hybrid simulation combining two space-time discretization of the discrete-velocity Boltzmann equation," Journal of Computational Physics, Vol. 349, 2017, pp. 399-414. https: //doi.org/10.1016/j.jcp.2017.08.029

[12] Shan, X., Yuan, X.-F., and Chen, H., "Kinetic theory representation of hydrodynamics: a way beyond the Navier-Stokes equation,” Journal of Fluid Mechanics, Vol. 550, 2006, pp. 413-441. https://doi.org/10.1017/S0022112005008153

[13] Mann, A., Pérot, F., Kim, M.-S., Casalino, D., and Fares, E., “Advanced Noise Control Fan Direct Aeroacoustics Predictions Using a Lattice-Boltzmann Method," 18th AIAA/CEAS Aeroacoustics Conference, 2012. https://doi.org/10.2514/6.2012-2287

[14] Sanjosé, M., Daroukh, M., de Laborderie, J., Moreau, S., and Mann, A., "Tonal fan noise prediction and validation on the ANCF configuration,” Noise Control Engineering Journal, Vol. 63, No. 6, 2015, pp. 552-562. https://doi.org/10.3397/1/376349

[15] Orselli, R. M., Carmo, B. S., and Queiroz, R. L., "Noise predictions of the advanced noise control fan model using lattice Boltzmann method and Ffowcs Williams-Hawkings analogy," Journal of the Brazilian Society of Mechanical Sciences and Engineering, Vol. 40, 2018, p. 34. https://doi.org/10.1007/s40430-018-0982-2.

[16] Gonzalez-Martino, I., and Casalino, D., "Fan Tonal and Broadband Noise Simulations at Transonic Operating Conditions Using Lattice-Boltzmann Methods,” 2018 AIAA/CEAS Aeroacoustics Conference, 2018. https://doi.org/10.2514/6.2018-3919.

[17] Loew, R. A., Lauer, J. T., McAllister, J., and Sutliff, D. L., “The Advanced Noise Control Fan,” 25th AIAA Aerodynamic Measurement Technology and Ground Testing Conference, 2006. https://doi.org/10.2514/6.2006-3150

[18] Hughes, C. E., Jeracki, R. J., Woodward, R. P., and Miller, C. J., "Fan Noise Source Diagnostic Test - Rotor Alone Aerodynamic Performance Results,” 8th AIAA/CEAS Aeroacoustics Conference, 2002. https://doi.org/10.2514/6.2002-2426

[19] Ffowcs Williams, J. E., and Hawkings, D. L., "Theory relating to the noise of rotating machinery," Journal of Sound and Vibration, Vol. 10, No. 1, 1969, pp. 10-21. https://doi.org/10.1016/0022-460X(69)90125-4.

[20] Sutliff, D. L., "Rotating rake turbofan duct mode measurement system," The Journal of the Acoustical Society of America, Vol. 118, 2005, p. 1864. https://doi.org/10.1121/1.4779000

[21] Bozak, R. F., “Advanced Noise Control Fan: Aerodynamic Performance,” Tech. Rep. NASA/TM-2009-215807, 2009.

[22] McAllister, J., Loew, R. A., Lauer, J. T., and Sutliff, D. L., "The Advanced Noise Control Fan Baseline Measurements,” 47th AIAA Aerospace Sciences Meeting, 2009. https://doi.org/10.2514/6.2009-624

[23] Bhatnagar, P. L., Gross, E. P., and Krook, M., “A Model for Collision Processes in Gases. I. Small Amplitude Processes in Charged and Neutral One-Component Systems," Physical Review, Vol. 94, No. 3, 1954, pp. 511-525. https://doi.org/10.1103/ PhysRev.94.511

[24] Coreixas, C., "High-order extension of the recursive regularized lattice Boltzmann method," Ph.D. thesis, Institut National Polytechnique de Toulouse, 2018. 
[25] He, X., Shan, X., and Doolen, G. D., "Discrete Boltzmann equation model for nonideal gases," Physical Review E, Vol. 57, No. 1, 1998, pp. R13-R16. https://doi.org/10.1103/PhysRevE.57.R13

[26] Grad, H., "Note on N-Dimensional Hermite Polynomials," Communications on Pure and Applied Mathematics, Vol. 2, No. 4, 1949, pp. 325-330. https://doi.org/10.1002/cpa.3160020402

[27] Hainaut, T., Le Garrec, T., Polacsek, C., Mincu, D. C., and Deck, S., “Aerodynamic and Aeroacoustic Numerical Investigation of an Axial Fan using Lattice Boltzmann Methods," 2018 AIAA/CEAS Aeroacoustics Conference, 2018. https://doi.org/10. 2514/6.2018-3922

[28] Lévêque, E., Toschi, F., Shao, L., and Bertoglio, J. P., "Shear-improved Smagorinsky model for large-eddy simulation of wall-bounded turbulent flows," Journal of Fluid Mechanics, Vol. 570, 2007, pp. 491-502. https://doi.org/10.1017/ S0022112006003429

[29] Meldi, M., Vergnault, E., and Sagaut, P., “An arbitrary Lagrangian-Eulerian approach for the simulation of immersed moving solids with Lattice Boltzmann Method,” Journal of Computational Physics, Vol. 235, 2013, pp. $182-198$. https://doi.org/10.1016/j.jcp.2012.10.014

[30] Chima, R. V., "Viscous Three-Dimensional Calculations of Transonic Fan Performance,” Tech. Rep. NASA/TM-103800, 1991.

[31] Tweedt, D. L., and Chima, R. V., "Rapid Numerical Simulation of Viscous Axisymmetric Flow Fields," 34th Aerospace Sciences Meeting and Exhibit, 1996. https://doi.org/10.2514/6.1996-449

[32] Goldstein, M. E., Aeroacoustics, McGraw-Hill Inc., 1976.

[33] Cantrell, R. H., and Hart, R. W., "Interaction between Sound and Flow in Acoustic Cavities: Mass, Momentum, and Energy Considerations," The Journal of the Acoustical Society of America, Vol. 36, No. 4, 1964, pp. 697-706. https: //doi.org/10.1121/1.1919047

[34] Daroukh, M., Moreau, S., Gourdain, N., Boussuge, J.-F., and Sensiau, C., "Effect of Distortion on Turbofan Tonal Noise at Cutback with Hybrid Methods," International Journal of Turbomachinery, Propulsion and Power, Vol. 2, No. 3, 2017 , p. 16. https://doi.org/10.3390/ijtpp2030016

[35] Tyler, J. M., and Sofrin, T. G., “Axial Flow Compressor Noise Studies,” Society of Automotive Engineers Transactions, Vol. 70, 1962, pp. 309-332. https://doi.org/10.4271/620532 